\title{
Shock temperature and melting in iron sulfides at core pressures
}

\author{
William W. Anderson ${ }^{1}$ and Thomas J. Ahrens \\ Lindhurst Laboratory of Experimental Geophysics, Seismological Laboratory \\ Califormia Institute of Technology, Pasadena
}

\begin{abstract}
The temperatures of shock-compressed $\mathrm{FeS}$ and $\mathrm{FeS}_{2}$ in the pressure ranges 125-170 $\mathrm{GPa}$ and 100-244 GPa, respectively, are reported and used to constrain the melting curves and thermodynamic properties to core pressures. A fit of the Lindemann law parameters corresponding to the usual functional form for the lattice Grüneisen parameter gives $\gamma_{L}=1.17 \pm 0.13$ and $n_{L}=$ $0.5 \pm 0.5$ for the high-pressure phase of FeS at $\rho=5340 \mathrm{~kg} / \mathrm{m}^{3}$ and $\gamma_{L}=2.18 \pm 0.32$ and $n_{L}=$ $1.6 \pm 0.7$ for $\mathrm{FeS}_{2}$ at $\rho=5011 \mathrm{~kg} / \mathrm{m}^{3}$. The entropies of fusion are $203 \mathrm{~J} \mathrm{~kg}^{-1} \mathrm{~K}^{-1}$ for FeS at 120 $\mathrm{GPa}$ and $\sim 180 \mathrm{~J} \mathrm{~kg}^{-1} \mathrm{~K}^{-1}$ for $\mathrm{FeS}_{2}$ at $220 \mathrm{GPa}$. We find that the melting temperature of $\mathrm{FeS}$ is $3240 \pm 200 \mathrm{~K}, 4210 \pm 700 \mathrm{~K}$, and $4310 \pm 750 \mathrm{~K}$ at $136 \mathrm{GPa}, 330 \mathrm{GPa}$, and $360 \mathrm{GPa}$, respectively. For $\mathrm{FeS}_{2}$, the melting temperatures are $3990 \pm 300 \mathrm{~K}, 5310 \pm 700 \mathrm{~K}$, and $5440 \pm 750 \mathrm{~K}$, respectively, for the same pressures. The electronic specific heat for $\mathrm{FeS}$ is given by $C_{e}=\beta_{0}(\rho / \rho)^{\gamma_{e}}$ with $\beta_{0}=0.25 \pm 0.10 \mathrm{~J} \mathrm{~kg}^{-1} \mathrm{~K}^{-2}$ and $\gamma_{e}=1.34$ for $\rho_{0}=5340 \mathrm{~kg} / \mathrm{m}^{3}$ for the highpressure solid phase and $\beta_{0} \approx 0.05 \mathrm{~J} \mathrm{~kg}^{-1} \mathrm{~K}^{-2}$ and $\gamma_{e}=1.34$ for $\rho_{0}=5150 \mathrm{~kg} / \mathrm{m}^{3}$ for the liquid phase. For $\mathrm{FeS}_{2}$, there is no detectable electronic contribution, and the lattice specific heat is only $67 \%$ of the Dulong-Petit limit, possibly implying tight $S-S$ binding in $S_{2}$ units. A reexamination of all shock wave melting data for $\mathrm{Fe}$ indicates these approximately agree, but they do not resolve the disagreement between the extrapolated static diamond anvil cell data sets. Fe should melt at $-6600 \mathrm{~K}$ at $243 \mathrm{GPa}$ and $6900 \pm 750 \mathrm{~K}$ at $330 \mathrm{GPa}$ (the pressure of the inner coreouter core boundary). Because the FeS melting curve falls well below that of $\mathrm{FeS}_{2}$, FeS may eventually undergo peritectic melting at high pressures, while $\mathrm{FeS}_{2}$ melts congruently.
\end{abstract}

\section{Introduction}

Sulfur is believed to be a light component of the predominantly iron core of the Earth because of its cosmic abundance and ability to dissolve readily into liquid iron [Murthy and Hall, 1972; Usselman, 1975a,b; Brett and Bell, 1969]. Arguments against a significant amount of sulfur in the core based on depletions of less volatile lithophile elements in mantle xenoliths [Ringwood, 1977; Ringwood and Kesson, 1977] assume that lithophile and siderophile elements have similar fates during formation and evolution of the earth and are open to argument. Because many models of core formation [e.g., Ruff and Anderson, 1980, Stevenson, 1981, Brett, 1984] show that inclusion of available sulfur is almost unavoidable due to its solubility in liquid $\mathrm{Fe}$ and depression of the $\mathrm{Fe}$ liquidus, an understanding of the thermodynamics of the Fe-S system is important to the study of core formation and evolution.

Early models of phase relationships in the Fe-S system under core conditions were based on extrapolation from relatively low-pressure data (see, e.g., Usselman [1975a,b] and Anderson et al. [1987]). Advent of the diamond anvil high-pressure cell has significantly extended the range of experimental studies of melting in Fe [Boehler, 1986;

\footnotetext{
${ }^{1}$ Now at Department of Geology and Physics, Georgia Southwestern College, Americus.

Copyright 1996 by the American Geophysical Union.

Paper number $95 \mathrm{JB} 01972$.

$0148-0227 / 96 / 95 \mathrm{JB}-01972 \$ 05.00$
}

Williams et al., 1987; Boehler et al., 1990; Boehler, 1993; Saxena et al., 1994] and compositions in the Fe-S system [Williams and Jeanloz, 1990; Boehler, 1992], but the highest pressures at which melting can be observed presently are achieved via shock compression [Williams et al., 1987; Bass et al., 1987; Tan and Ahrens, 1990; Ahrens et al., 1990a,b; Yoo et al., 1993]. Here, we use shock temperature measurements to extend the melting curves of $\mathrm{FeS}$ and $\mathrm{FeS}_{2}$ to core pressures.

\section{Experimental Techniques}

The experimental techniques used in the present study have been discussed in detail elsewhere [Lyzenga and Ahrens, 1979; Kondo and Ahrens, 1983; Schmitt and Ahrens, 1983; Boslough, 1984; Bass et aL, 1987; Tan and Ahrens, 1990; Ahrens et al., 1990a]. A sample of $\mathrm{FeS}$ or $\mathrm{FeS}_{2}$ was sandwiched between a metal driver plate and a transparent window to form a target assembly. In all but one experiment, the window was a single crystal of $\mathrm{Al}_{2} \mathrm{O}_{3}$. The one exception used a single crystal of LiF. The target assembly was placed in a chamber evacuated to -0.1 torr and impacted by a gun-accelerated projectile consisting of a polycarbonate sabot and a metal flyer plate (Figure 1).

During the experiment, the sample-window interface was observed through the transparent window. Thermal radiation from the sample-window interface was directed by an expendable mirror into a four-color pyrometer or a grating spectrometer and the resulting signals were recorded on oscilloscopes and a 100-MHz digital recorder or, in the case of the spectrometer, a multichannel analyzer. 


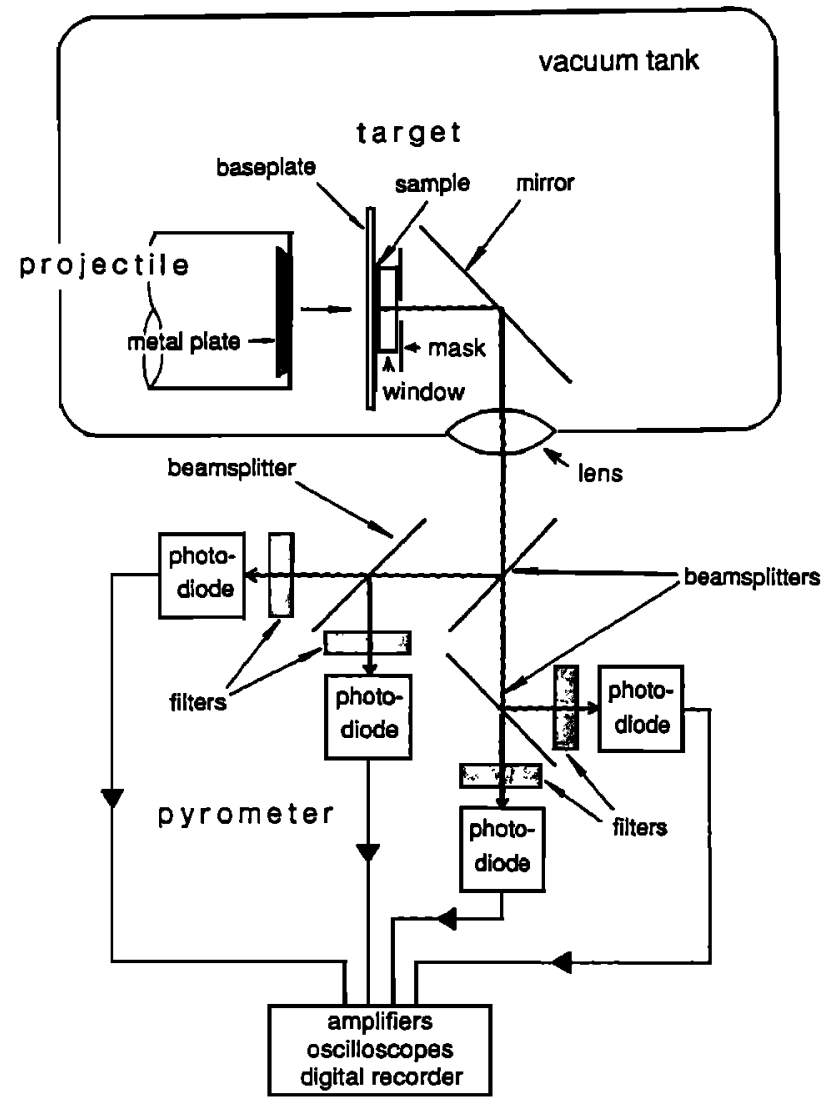

Figure 1. Diagram of a typical shock temperature experiment. The configuration shown is for the fourchannel pyrometer, which uses a series of beamsplitters to direct radiation from the sample-window interface into the four detectors.

Most of the samples were optically thick films vapordeposited directly on the windows [Bass et al., 1987]. Examination of the films via optical and scanning electron microscopy showed the samples to be nonporous at micrometer and submicrometer scales. The film thickness was generally of the order of $1 \mu \mathrm{m}$, as determined by direct measurement of broken films viewed edge on with the electron microscope. The compositions of the films (Table 1) were also determined via quantitative energy dispersive $X$ ray spectrometry and $X$ ray fluorescence spectrometry. The FeS films used were stoichiometic with no detectable contaminants, while the $\mathrm{FeS}_{2}$ films showed minor $\mathrm{Zn}$ contamination. The $\mathrm{Fe} / \mathrm{S}$ ratio in the $\mathrm{FeS}_{2}$ also seems to be somewhat Fe-rich compared to stoichiometric $\mathrm{FeS}_{2}$, but the precision of the analyses and uncertainties in $X$ ray absorption corrections applied to the thin film data make this conclusion questionable. We should note that obtaining usable thin film samples proved extremely difficult, especially in the case of FeS. The difficulty was in obtaining the correct stoichiometry. Most attempts at obtaining $\mathrm{FeS}$ resulted in the production of $\mathrm{FeS}_{2}$. Additionally, the differential thermal expansion of the film and window materials caused the films to peel away from the window if the film and window were exposed to thermal cycling. The crystallinity of the FeS films was confirmed by $X$ ray diffraction (Figure 2 ), which showed that the sample is crystalline troilite or pyrrhotite, with no detectable amorphous material. The $\mathrm{FeS}_{2}$ films were not X rayed but were deposited at the same temperature condition as the FeS and produced shock temperature data consistent with a sample of known crystalline material, so that these films are sufficiently crystalline to be indistinguishable from pyrite. One experiment (217) used a single-crystal slab of natural pyrite with an Archimedian density of $4.945 \pm .002 \mathrm{Mg} / \mathrm{m}^{3}$. The slab was ground flat to a thickness of $2.633 \mathrm{~mm}$, and the surface contacting the window was polished until the surface visible during the experiment deviated by $\leq 0.25 \mu \mathrm{m}$ from the shape of the window surface.

Before each experiment, the entire optical path and pyrometer response was calibrated with a standard lamp (Optronics Laboratories model $220 \mathrm{H}$ ) placed in the position which is occupied by the target during the experiment. The light from the lamp was directed along the same optical path as the light emitted by the sample during the experiment and was interrupted at regular intervals by a mechanical chopper, allowing the absolute response of each pyrometer channel to be determined.

The experiment designated 723 was fundamentally different from the others in several respects. In this experiment, a powdered sample of natural pyrthotite with a composition of $\mathrm{Fe}_{88} \mathrm{~S}$ was compressed to a porosity of $31 \%$ (2.177 $\mathrm{mm}$ thick, $\rho_{0}=3.168 \mathrm{Mg} / \mathrm{m}^{3}$ ) and placed between an iron driver plate and a window made of LiF. The thermal radiation emitted by the sample during the experiment was directed into a grating spectrometer and was integrated by an optical multichannel analyzer for 300 ns to give a single spectrum [Kondo and Ahrens, 1983].

\section{Experimental Results and Data Analysis}

Impact of the flyer on the driver plate generates a planar shock wave which propagates into the target assembly. Because strong shock waves characteristically cause significant increases in entropy, shock-compressed materials achieve very high temperatures $\left(10^{3}-10^{4} \mathrm{~K}\right)$, with more compressible materials and those with lower specific heats being driven to higher temperatures. Since the window remains transparent upon passage of the shock wave [Wise and Chhabildas, 1986; Ahrens et al., 1990b; McQueen and Isaak, 1990], the sample-window interface acts as a visible surface which emits radiation according to the Planck function:

$$
I(\lambda, T)=\frac{\varepsilon c_{1}}{\lambda^{5}\left(e^{c_{2} / \lambda T}-1\right)}
$$

where $\lambda$ is the wavelength, $T$ is the temperature, $c_{1}=$ $5.95522 \times 10^{-17} \mathrm{~W} \mathrm{~m}^{2} / \mathrm{sr}, c_{2}=1.4388 \times 10^{-2} \mathrm{~m} \cdot \mathrm{K}$, and $\varepsilon$ is the emissivity (which may depend on $\lambda$ ). Kondo [1994] disputes the transparency of shocked $\mathrm{Al}_{2} \mathrm{O}_{3}$ and suggests that nonthermal radiation from shock-induced defects is

Table 1. Chemical Analyses of Thin Film Samples

\begin{tabular}{cccc}
\hline Sample & $\mathrm{Fe} / \mathrm{S}$ & $\mathrm{Cr} / \mathrm{S}$ & $\mathrm{Zn} / \mathrm{S}$ \\
\hline $\mathrm{FeS}$ & 1.0 & --- & $\cdots$ \\
$\mathrm{FeS}_{2}$ & 0.593 & 0.001 & 0.024 \\
\hline
\end{tabular}




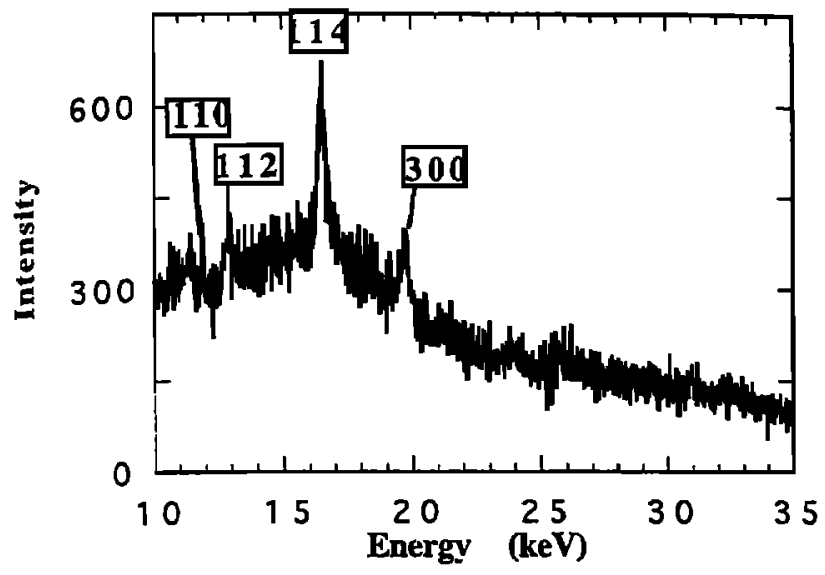

Figure 2. $\mathrm{X}$ ray diffraction pattern for FeS film taken in energy dispersive mode using white radiation on Beam Line X17C, National Synchrotron Light Source, Brookhaven National Laboratory. Diffraction peaks that may be indexed either as troilite or pyrrhotite.

significant. While we do not dispute those results in principle, the data we present will demonstrate that for the present study, this effect is not significant. This point will be further discussed later.

The instrumental records were converted to temperatures by minimization of the function

$$
\chi^{2}=\sum\left[\frac{V_{o b s, i}-\Theta_{i} A_{s} J_{0}^{\infty} I\left(\lambda_{s} T\right) R_{i}(\lambda) d \lambda}{\sigma_{o b s, i}}\right]^{2},
$$

where $V_{o b s, i}$ is the observed voltage from the $i$ th pyrometer channel, $R_{i}(\lambda)$ is the spectral response (i.e., signal resulting from unit irradiance at a given wavelength) of channel $i, \theta_{l}$ is the solid angle subtended by the active surface of the detector for channel $i$, as viewed from the emitting surface of the sample-window interface, and $A_{s}$ is the visible area of the emitting surface. Figure 3 shows the response $R$ of the four pyrometer channels as a function of wavelength for a typical experiment. The time response characteristics of the pyrometer for use in deconvolving the instrument response from the data at different gain settings (Figure 4) were measured using a light pulse generator. The values of $R_{i}(\lambda)$ used for the data reduction include the effects of the optical components in the system and internal reflections in the window [Born and Wolf, 1980, chapter 1].

Previous studies of temperatures in shock-compressed materials assumed wavelength-independent emissivity in the reduction of radiance data to give temperatures. In the present case, this assumption could be seriously in error as can be seen in Figure 5. We chose to apply two models for $\varepsilon$. The first is that of constant, or gray, emissivity. The second assumes that the emissivity of a material at high pressure is proportional to that of the same material at STP. In reality, the emissivity behavior should fall somewhere between these two end-member cases, because the effect of compression on metallic and semimetallic compounds is to broaden electronic energy bands. This broadening results in smearing out of spectral features so that the spectrum becomes much flatter. There is the possibility that narrow spectral features could appear due to shock-induced defects. This may be the origin of some of the misfit that will be seen in the radiance fits. However, the strong dependence of $I(\lambda)$ on $T$ requires that the fit temperature be close to the true result for any reasonable values of $\varepsilon$.

During the fitting process, both $T$ and a constant multiplicative scaling factor for $\varepsilon$ were allowed to vary. With the thin films, the earliest usable datum after the shock arrived at the sample-window interface was used, generally 10 to $20 \mathrm{~ns}$ after arrival of the shock wave at the interface. This was done to avoid complications arising from heat being conducted through the sample from the driver-sample interface. It also minimizes the optical path length through shocked window, which is important since the optical properties of the shocked window are not wellknown.

Projectile impact velocities were determined using timeseparated flash X-radiography. The pressures, densities, and internal energies achieved during the experiments were determined from the measured impact velocities of the projectiles via the impedance matching technique [Walsh et al., 1957] using shock Hugoniot curves described by

$$
U_{s}=C_{0}+s u_{p}
$$

where $U_{s}$ and $u_{p}$ are the shock wave velocity and the particle velocity of the shocked material, both determined in the rest frame of the unshocked material. Table 2 lists values of $\rho_{0}$, $C_{0}$, and $s$ used for this study. Because of the differences in the properties of the sample and window, a shock or release wave is reflected from the sample-window interface. Since most of the samples are less than $0.01 \mathrm{~mm}$ thick after the initial shock, multiple reflections occur which bring the sample from the initial shock pressure $P_{H}$ to the final pressure $\boldsymbol{P}_{f}$ given by the impedance match appropriate for the driver plate impacting the window, on a timescale of $<10$ ns. Since these reverberations are approximately isentropic and typically involve pressure changes small compared to $P_{H}$, we approximate the $P-V$ path of the reverberation in the sample by the $P \cdot V$ projection of the

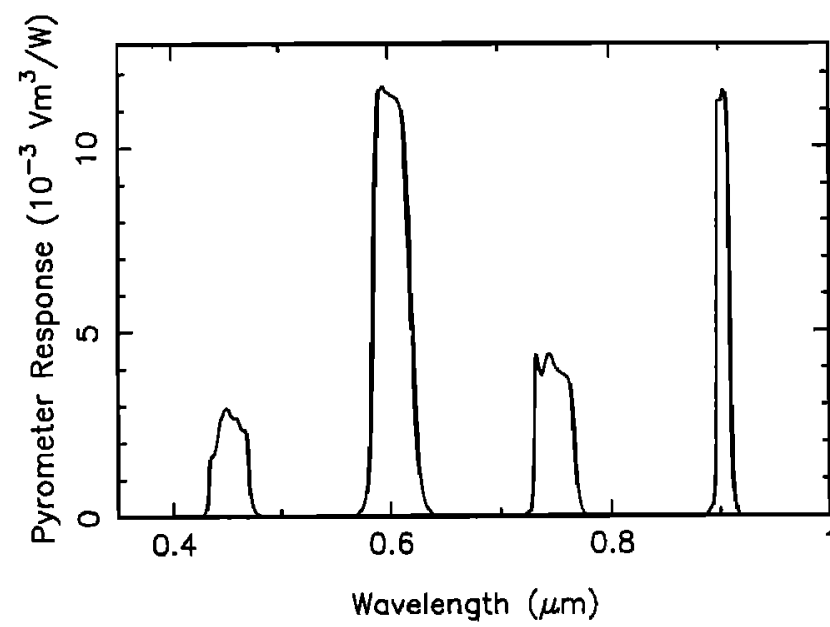

Figure 3. Spectral response of the four channels of the pyrometer used for this study, determined by combination of calibration voltages with spectral characteristics of the optical components and photodiodes in the system, as measured or provided by the component manufacturers. 


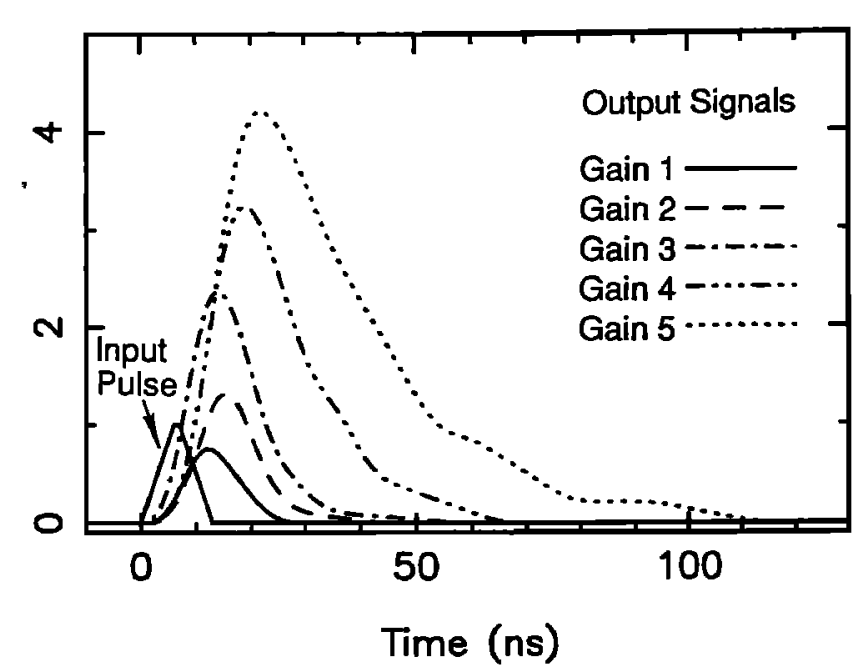

Figure 4. Time response for a typical pyrometer channel. The (labeled) "input pulse" (of uncalibrated intensity) from the light pulse generator and the accompanying curves are output signals arising from convolution of the pyrometer time response with the input signal. An increase of 1 in gain represents a factor of 2 increase in the level of a dc signal.

sample Hugoniot curve, which is similar to the isentrope over a restricted enough range.

Table 3 and Figures 6 and 7 present the results from the experiments. Based on the overall goodness of fit, the FeS data are better modeled using an emissivity proportional to the 1-bar FeS absorption (which is the same as the 1 bar emissivity for optically thick samples), while $\mathrm{FeS}_{2}$ is better modeled as a gray emitter. The stated uncertainties are the square roots of the formal variances, which were determined during the fitting process. The uncertainties in $T$ seem low, given the error bars on the data seen in Figure 5, but it must be remembered that very small variations in $T$ cause large deviations in the overall radiance. For reasonable values of $\varepsilon$, the error bars do admit very small uncertainties in the fit $T$. In most cases, the uncertainties on $T$ are larger than the variation resulting the choice of emissivity model. The one major exception is for shot 206, where the fit value of $T$ varies by almost $900 \mathrm{~K}$ depending on the emissivity model chosen. Hence the temperature for this one particular shot must be considered to be very uncertain.

In our experiments, the samples were more compressible and had lower specific heats than the windows and thus were driven to much higher temperatures than the windows. In such a case, the temperature at the interface is controlled by the diffusion of heat from the sample into the window. To obtain temperatures that are characteristic of the samples alone, we use the models of Urtiew and Grover [1974], Grover and Urtiew [1974], and Tan and Ahrens [1990] to relate the sample-window interface temperature to the temperature in the interior of the sample using the diffusion equation [Carslaw and Jaeger, 1959]. These models are case-specific, based on whether the Hugoniot falls on the melting transition or whether heat transfer results in the initiation of phase changes in the sample or window, and relate the interface temperature to either the melting temperature or the Hugoniot temperature of the sample.

The occurrence of melting (or other highly endothermic changes) can be detected in the data set as a whole based on its effect on the $P-T$ trends of the data. Endothermic phase changes buffer the temperature rise along a Hugoniot curve because of latent heat effects. As a consequence, the Hugoniot curve demonstrates a kink where it intersects a phase boundary in $P-T$ space, coinciding with the phase boundary until sufficient energy is available in the Hugoniot state to drive the phase transition to completion [Bass et al., 1987].

Data for both $\mathrm{FeS}$ and $\mathrm{FeS}_{2}$ show a series of points falling on trends of very slowly increasing temperature with increasing pressure. The highest-pressure points in both cases fall above these trends and, for $\mathrm{FeS}_{2}$, the lowestpressure datum falls below the trend. The points falling on the shallow slope represent an endothermic phase change with a large latent heat [Tan and Ahrens, 1990]. Based on the work of Brown et al. [1984] for FeS, and Ahrens and Jeanloz [1987] for $\mathrm{FeS}_{2}$, we conclude that those points indicate melting behavior, while the lowest-pressure point in $\mathrm{FeS}_{2}$ is in the solid phase and the highest-pressure points in both materials are in the liquid phases.

If the reverberated state in the sample falls on the melting curve or if heat transfer from the sample to the window initiates freezing in the sample from a completely liquid reverberated state, then we solve for the melting temperature at the reverberated or released pressure. Otherwise, we obtain a reverberated or released state temperature and can transform this along an (approximately) isentropic reverberation or release path to obtain the Hugoniot temperature.

The final state of the sample represented by the values of $\boldsymbol{P}_{f}$ and the interface temperatures in Table 3 may be solid, liquid, or a mixture of solid and liquid, depending on the actual temperature of the sample relative to its melting temperature. The Hugoniot temperature of the window, however, falls well below the window melting curve, based on the estimated melting curves of the window materials [Ahrens et al., 1990a] and the internal energies along the Hugoniot curves of the window materials. We can treat the thermal diffusion between the sample and window as onedimensional because we used a mask to exclude all but the center of the sample-window interface from observation.

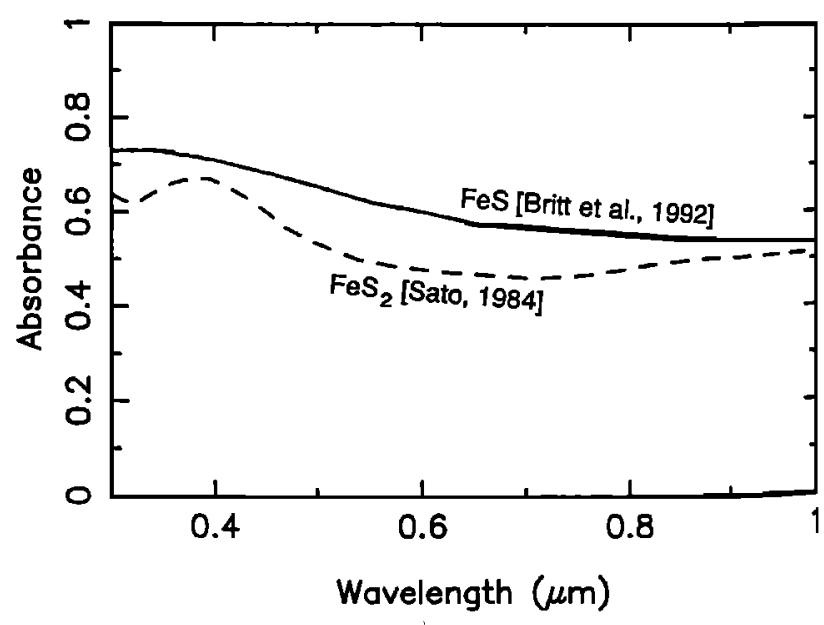

Figure 5. Spectral absorbance of $\mathrm{FeS}$ and $\mathrm{FeS}_{2}$ at 1 bar. For optically thick samples, emissivity and absorptivity are equal. 
Table 2. Shock Hugoniot Parameters

\begin{tabular}{ccccc}
\hline Material & $\rho_{0}, \mathrm{Mg}^{3} \mathrm{~m}^{3}$ & $C_{0}, \mathrm{~km} / \mathrm{s}$ & $s$ & Reference \\
\hline $\mathrm{W}$ & 19.235 & 4.040 & 1.23 & 1 \\
$\mathrm{Ta}$ & 16.650 & 3.293 & 1.307 & 2 \\
$\mathrm{Fe}$ & 7.850 & 3.955 & 1.58 & 3 \\
$\mathrm{Al}$ & 2.712 & 5.380 & 1.34 & 1 \\
$\mathrm{Cu}$ & 8.930 & 3.940 & 1.489 & 1 \\
$\mathrm{FeS} \mathrm{(liquid)}$ & 4.829 & 2.947 & 1.578 & 4 \\
$\mathrm{FeS}$ (solid) & 4.829 & 3.865 & 1.351 & 4 \\
$\mathrm{FeS}_{2}$ & 5.011 & 5.478 & 1.401 & 5 \\
$\mathrm{Al}_{2} \mathrm{O}_{3}$ & 3.986 & 8.740 & 0.957 & 6 \\
$\mathrm{LiF}$ & 2.640 & 5.148 & 1.353 & 7 \\
\hline
\end{tabular}

References: (1) Marsh [1980]; (2) Mitchell and Nellis [1981]; (3) Brown and McQueen [1986]; (4) Brown et al. [1984]; (5) Ahrens and Jeanloz [1987]; (6) Erskine [1994]; (7) Carter [1973].

The simplest case, which we call model 0 , occurs when two conditions are met: (1) no phase changes occur because of heat transfer between the sample and the window and (2) the final state of the sample does not fall into the mixed phase region. The final state of the sample may be either liquid or solid, as long as it is a single phase. The samplewindow interface temperature $T_{I}$ is given by [Urtiew and Grover, 1974; Grover and Urtiew, 1974]:

$$
\begin{gathered}
T_{I}=T_{s}-\frac{T_{s}-T_{w}}{I+\alpha} \\
\alpha=\left(\frac{K_{s} \rho_{s} C_{s}}{K_{w} \rho_{w} C_{w}}\right)^{I / 2}
\end{gathered}
$$

where $T_{s}$ and $T_{w}$ are the sample and window temperatures away from the interface and $K$ and $C$ are the thermal conductivity and specific heat, respectively, and the subscripts $s$ and $w$ refer to the sample and window.

Models I, II, and III of Tan and Ahrens [1990] treat the complications arising from phase changes in the sample and window. Model I considers a freezing front propagating into the sample from the interface, due to conduction of heat into the window and gives

$$
T_{I}=T_{w}+\frac{\alpha\left(T_{m s}-T_{w}\right)}{\alpha-\operatorname{erf} \mu}
$$

where $T_{m, s}$ is the sample melting temperature and $\mu$ is related to the propagation rate of the freezing front. If we assume that the molten and solid sample have similar thermal properties and densities, then $\mu$ is the root of the equation

$$
\begin{aligned}
\frac{T_{s}-T_{m, s}}{l+\operatorname{erf} \mu} e^{-\mu^{2}} & -\frac{\alpha^{2}\left(T_{m, s}-T_{w}\right)}{\alpha-\operatorname{erf} \mu} e^{-\mu^{2}} \\
& -\frac{\pi^{1 / 2} \chi_{s} \Delta H_{m, s} \mu}{C_{s}}=0,
\end{aligned}
$$

where $\chi_{s}$ is the fraction of sample melted by the shock and reverberation process prior to any conduction-induced freezing and $\Delta H_{m s}$ is the enthalpy of melting of the sample.
When $0<\chi_{s}<1, T_{s}=T_{m, s}$ and the first term in (7) vanishes. There is a range of cases in which $\chi_{s}=1$, but for which this model applies because sufficient heat is conducted into the window to induce freezing in the sample.

Model II treats the reverse problem from model I, i.e., conduction-induced melting in the window but no phase change in the sample. In this case,

$$
T_{I}=T_{s}+\frac{\alpha\left(T_{s}-T_{m, w}\right)}{I+\alpha \operatorname{erf} \lambda},
$$

where $T_{m w}$ is the melting temperature of the window and $\lambda$, which is analogous to $\mu$ in model $I$, is related to the rate of advance of the melting front in the window. Assuming similar properties between the liquid and solid window material, $\lambda$ is the root of

$$
\begin{gathered}
\frac{\alpha\left(T_{s}-T_{m, w}\right)}{I+\alpha \operatorname{erf} \lambda} e^{-\lambda^{2}}-\frac{T_{m, w}-T_{w}}{1-\operatorname{erf} \lambda} e^{-\lambda^{2}} \\
-\frac{\pi^{1 / 2} \Delta H_{m, w} \lambda}{C_{w}}=0
\end{gathered}
$$

Here, $\Delta H_{m w}$ is the enthalpy of melting of the window material.

Model III combines the effects treated in models I and II. In this case,

$$
T_{I}=T_{m, s}+\frac{\left(T_{m, s}-T_{m, w}\right) \operatorname{erf} \mu}{\alpha e r f \lambda-\operatorname{erf} \mu},
$$

where $\mu$ and $\lambda$ are the roots of the simultaneous equations

$$
\begin{gathered}
\frac{\alpha\left(T_{m s}-T_{m, w}\right)}{\alpha e r f \lambda-\operatorname{erf} \mu} e^{-\lambda^{2}}-\frac{T_{m, w}-T_{w}}{1-\operatorname{erf} \lambda} e^{-\lambda^{2}} \\
-\frac{\pi^{1 / 2} \Delta H_{m, w} \lambda}{C_{w}}=0 \\
\frac{T_{s}-T_{m, w}}{1+\operatorname{erf} \mu} e^{-\mu^{2}}-\frac{T_{m, s}-T_{m, w}}{\alpha e r f \lambda-\operatorname{erf} \mu} e^{-\mu^{2}} \\
-\frac{\pi^{1 / 2} \chi_{s} \Delta H_{m, s} \mu}{C_{s}}=0 .
\end{gathered}
$$




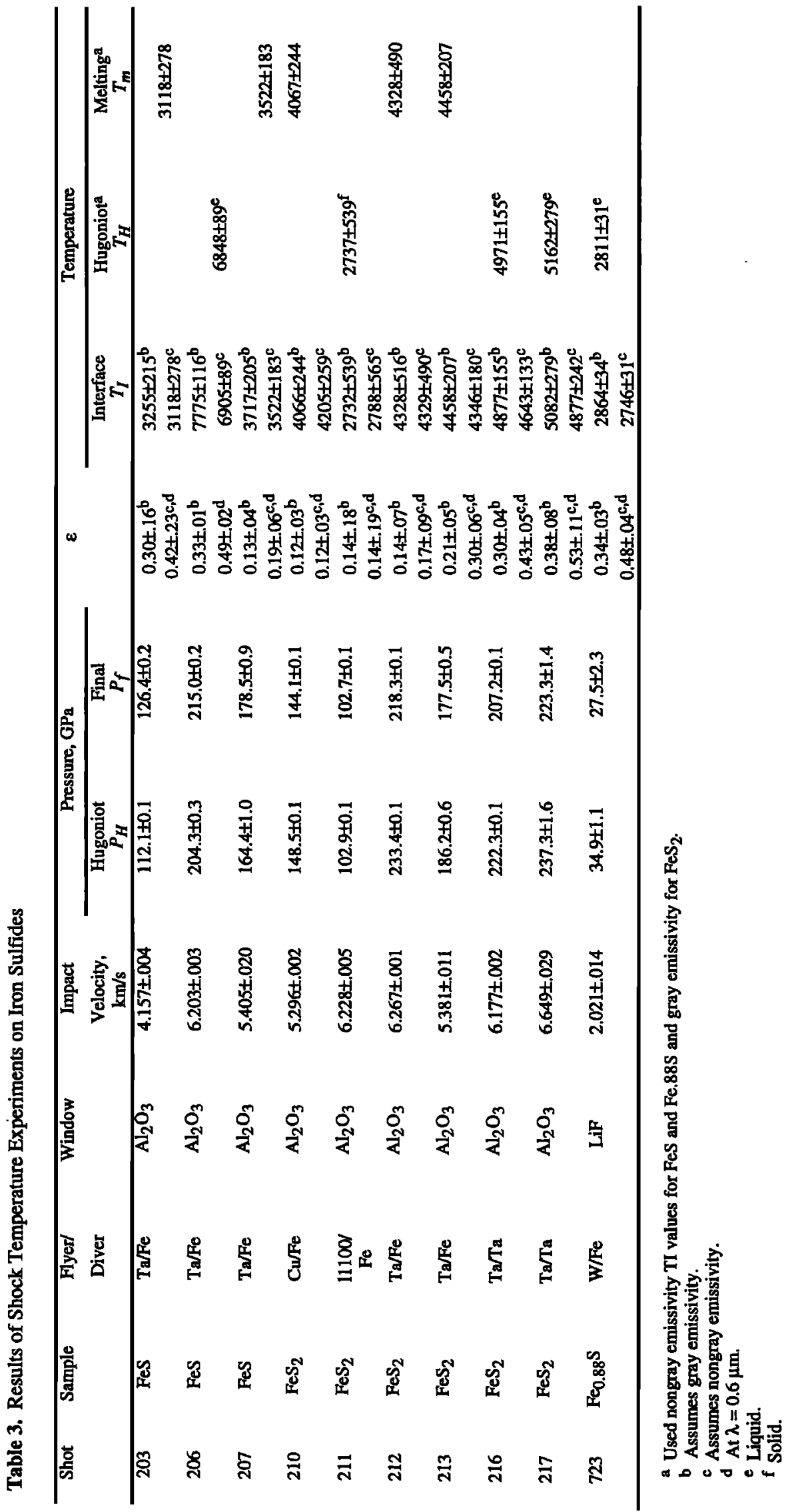



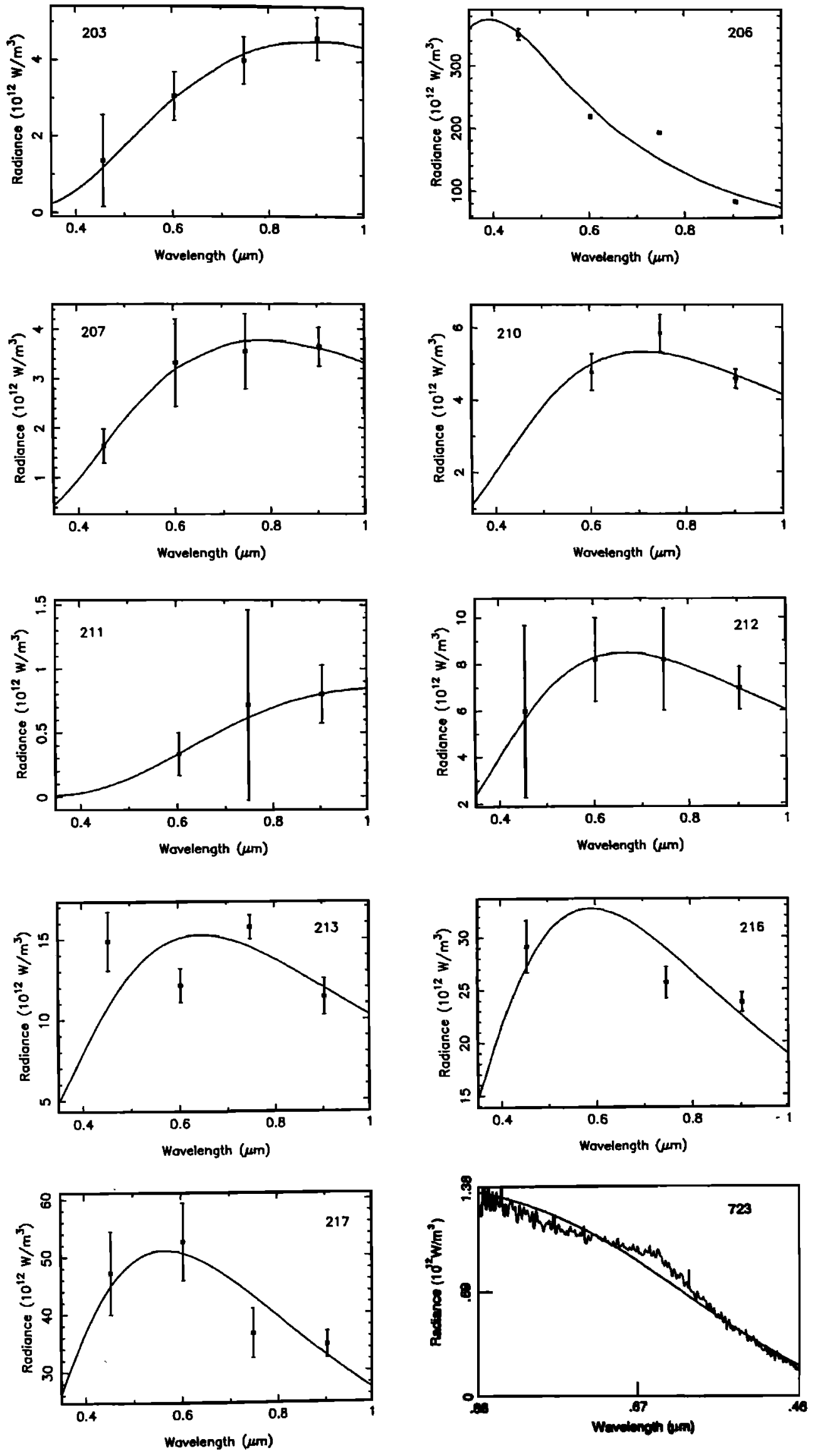

Figure 6. Spectral radiance curves fit to experimental data. 

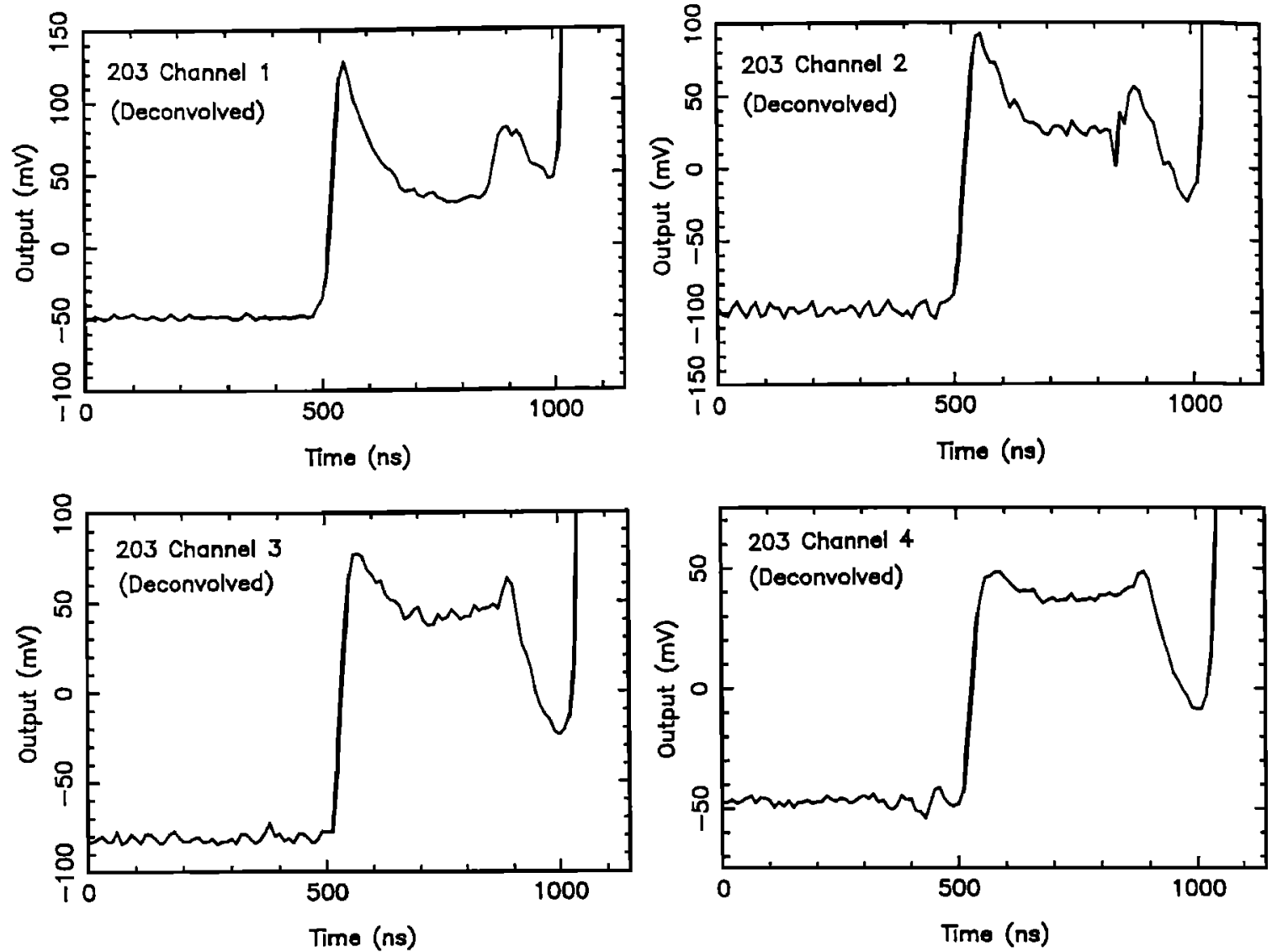

Figure 7. Deconvolved instrumental signals from shot 203. Wavelengths increase from channel 1 to channel 4 . The decay at shorter wavelengths may be due to effects in the shocked window or conduction through the sample. Shock arrival at the sample-window interface is at $t=510 \mathrm{~ns}$.

In the solutions for $T_{s}$ and $T_{m s}$, the equations are rearranged [Tan and Ahrens, 1990]:

Model 0

$$
T_{s}=T_{I}+\frac{T_{I}-T_{w}}{\alpha}
$$

Model I

$$
\begin{gathered}
T_{m, s}=T_{w}+\frac{\left(T_{I}-T_{w}\right)(\alpha-\operatorname{erf} \mu)}{\alpha} \quad\left(\chi_{s}<1\right) \\
T_{i} \leq T_{m, s} \leq T_{w}+\frac{\left(T_{I}-T_{w}\right)(\alpha-\operatorname{erf} \mu)}{\alpha} \quad\left(\chi_{s}=1\right)
\end{gathered}
$$

with $\mu$ being the root of (7).

Model II

$$
T_{s}=T_{I}+\frac{T_{I}-T_{m, w}}{\alpha e r f \lambda}
$$

with $\lambda$ being the root of (9).
Model III

$$
\begin{gathered}
T_{m, s}=T_{I}+\frac{\left(T_{m, w}-T_{I}\right) \operatorname{erf} \mu}{\alpha e r f \lambda} \quad\left(\chi_{s}<1\right) \\
T_{I} \leq T_{m, s} \leq T_{I}+\frac{\left(T_{m, w}-T_{I}\right) \operatorname{erf} \mu}{\alpha e r f} \quad\left(\chi_{s}=1\right)
\end{gathered}
$$

where $\lambda$ for model III is given by the root of

$$
\frac{T_{1}-T_{m, w}}{\operatorname{erf} \lambda} e^{-\lambda^{2}}-\frac{T_{m, w}-T_{w}}{1-\operatorname{erf} \lambda} e^{-\lambda^{2}}-\frac{\pi^{1 / 2} \Delta H_{m, w} \lambda}{C_{w}}=0
$$

and $\mu$ is given approximately by the root of

$$
\begin{gathered}
\frac{T_{I}-T_{m, w}}{\alpha e r f \lambda} e^{-\mu^{2}}+\frac{\pi^{1 / 2} \Delta S_{m, s} \chi_{s} \mu}{C_{s}} \\
\cdot\left[T_{I}-\frac{\left(T_{I}-T_{m, w}\right) \operatorname{erf} \mu}{\alpha e r f \lambda}\right]=0 .
\end{gathered}
$$


Now, we have replaced $\Delta H_{m s}$ by $T_{m s} \Delta S_{m s}$ where $\Delta S_{m s}$ is the entropy change of the sample upon melting. The relationship between $T_{I}$ and $T_{m s}$ cannot be determined uniquely for models I and III when $\chi_{s}=1$, because the initial temperature of the sample in the diffusive heat transfer solution is not known. Only the limits in (15) and (18) can be obtained. It should be noted that (17) and (20) are different from what should be the equivalent expressions of Tan and Ahrens [1990 equations (54) and (56), respectively]. This is due to typographical errors in the final version of their paper.

Which model applies is easily determined upon examination of the total data set as already discussed. The forms of (4)-(12) indicate that $T_{I}$ will be most sensitive to the thermal state of the material with the greater thermal conductivity, which is the sample in the present case. The shallow trends in the data for $T_{I}$ thus reflect similar trends in the temperature of the sample. Specifically, these points represent cases where conductive freezing of the sample (i.e., models I and III) occurs. Whether melting occurs in the window is simply determined by comparison of $T_{I}$ with $T_{m w}$, where $T_{I} \geq T_{m w}$ indicates melting of the window.

In order to obtain values of $T_{s}$ or $T_{m s}$ from $T_{l}$, we need estimates for the thermal properties of the materials involved. For the windows, we assume the Debye model for thermal conductivity of a dielectric [Kittel, 1966]:

$$
K=\frac{1}{3} C u \Lambda \text {, }
$$

where $C$ is heat capacity per unit volume, $u$ is sound speed, and $\Lambda$ is the phonon mean free path. $\Lambda$ is finite because of anharmonic multiple-phonon scattering processes, with the consequence that $A$ depends on some inverse power of the phonon number density which, in turn, is roughly proportional to $T$. Hence $K \sim T^{-q}$. Specifically, only threephonon scattering gives $q=1$, while only four-phonon scattering gives $q=2$, etc. Three-phonon processes are expected to dominate, so that $q$ should be close to, but somewhat larger than, 1 . We extrapolate $K$ at $10^{5} \mathrm{~Pa}(1$ bar) to the temperature of interest via

$$
K\left(T, 10^{5} \mathrm{~Pa}\right)=K_{o}\left(\frac{298}{T}\right)^{q} .
$$

Values of $K_{0}$ and $q$ are given in Table 4. To correct for compression, we use the Debye-Grüneisen model [Roufosse and Jeanloz, 1983]:

$$
\left(\frac{\partial \ln K}{\partial \ln p}\right)_{T}=\frac{2}{3}+3\left(\frac{\partial \ln u}{\partial \ln \rho}\right)_{T}-2\left(\frac{\partial \ln \gamma_{l}}{\partial \ln \rho}\right)_{T}
$$

where $\gamma_{l}$ is the lattice Grüneisen parameter, which for dielectrics is essentially the thermodynamic Grineisen parameter $\gamma$. We adopt the commonly used form

$$
\gamma=\gamma_{0}\left(\frac{\rho_{0}}{\rho}\right)^{n}
$$

where

$$
n=I+\delta_{T}-K_{T}^{\prime}+\left(\frac{\partial \ln C_{V}}{\partial \ln \rho}\right)_{T}
$$

$K_{T}^{\prime}$ is the pressure derivative of the isothermal bulk modulus $K_{T}$ and $\delta_{T}$ is defined by

$$
\delta_{T}=-\frac{1}{\alpha}\left(\frac{\partial \ln K_{T}}{\partial T}\right)_{P}
$$

$\left(\partial \ln C_{V} / \partial \ln \rho\right)_{T}$ can be obtained using either a Debye or Einstein model for the specific heat. For simplicity, we have adopted the Einstein model:

$$
C_{V}=\frac{3 R}{\mu}\left(\frac{\theta_{E}}{T}\right)^{2} \frac{e^{\theta_{E} / T}}{\left(e^{\theta_{E} / T}-1\right)^{2}}
$$

where $R$ is the ideal gas constant, $\mu$ is the mean atomic weight and, $\theta_{E}$ is the Einstein temperature. $\theta_{E}$, like other lattice characteristic temperatures, obeys

$$
\frac{d \ln \theta_{E}}{d \ln \rho}=\gamma_{l} \text {. }
$$

We make the usual assumption that $n$ is constant and fix it with the STP values of the parameters in (25). The sound speed $u$ is related to the Debye characteristic temperature $\Theta_{D}$ by

\begin{tabular}{|c|c|c|c|c|c|c|c|c|}
\hline Material & $\begin{array}{c}\rho_{0} \\
\mathrm{~kg} / \mathrm{m}^{3}\end{array}$ & $\gamma_{0}$ & $n$ & $\begin{array}{c}K_{0} \\
\mathrm{w}^{-1} \mathrm{~K}^{-1}\end{array}$ & $q$ & $\begin{array}{c}\boldsymbol{\theta}_{D} \\
\mathbf{K}\end{array}$ & $\begin{array}{c}\beta_{0} \\
J_{\mathbf{k g}^{-1}} \mathbf{K}^{-2}\end{array}$ & $\gamma_{e}$ \\
\hline$\gamma-\mathrm{Fe}$ & $8298^{\mathrm{a}}$ & $2.2^{b}$ & $1.62^{b}$ & $\cdots$ & -- & $385^{c}$ & $0.092^{d}$ & $1.34^{\mathrm{d}}$ \\
\hline $\mathrm{FeS}$ & $5340^{e}$ & $1.54^{\mathrm{e}}$ & $1 \mathrm{e}$ & -- & --- & $674^{f}$ & 0 & 0 \\
\hline $\mathrm{FeS}_{2}$ & 50118 & $1.56 \mathrm{~g}$ & 18 & -.- & -- & $705^{h}$ & 0 & 0 \\
\hline $\mathrm{Al}_{2} \mathrm{O}_{3}$ & $3986^{i}$ & $1.324 \mathrm{j}$ & $1.16 j$ & $46.95^{k}$ & $1.26^{\mathrm{k}}$ & $1025 \mathrm{j}$ & -- & ... \\
\hline LiF & $2640^{1}$ & $1.935 \mathrm{j}$ & $1.1 \mathrm{j}$ & $14.00^{\mathrm{m}}$ & $1.22^{\mathrm{m}}$ & $661^{j}$ & -- & -. \\
\hline $\begin{array}{l}\text { a Meo et } \\
\text { b Jeanlo } \\
\text { c Andren } \\
\text { d Boness } \\
\text { e Brown } \\
\text { f Svends } \\
\text { g Ahrens }\end{array}$ & $\begin{array}{l}\text { 1990]. } \\
\text { 79]. } \\
\text { 773]. } \\
\text { [1986]. } \\
\text { [1984]. }\end{array}$ & & & \multicolumn{5}{|c|}{$\begin{array}{l}\text { h Fit to tabulation from Chase et al. [1985]. } \\
\text { i Erskine [1994]. } \\
\text { j Estimated from tabulation of Sumino and Anderson [1984]. } \\
\text { k Fit to values tabulated by Touloukian et al. [1970]. } \\
\text { l Carter [1973]. } \\
\text { m Fit to measurements of Men' et al. [1974] and Petrov et al. [1974]. }\end{array}$} \\
\hline
\end{tabular}

$$
\Theta_{D} \propto u p^{1 / 3} .
$$

$\Theta_{D}$, like $\theta_{E}$, obeys (28), so that, for a dielectric,

$$
\frac{d \ln u}{d \ln \rho}=\gamma-\frac{1}{3} \text {. }
$$

This gives the compression correction to $K$ as

Table 4. Parameters Used in Thermal Diffusion Corrections 
Table 5. Equation of State Parameters Used to Obtain $P$ - $p-T$ Relations in This Study

\begin{tabular}{ccccccccccc}
\hline Material & $\rho_{0}$ & $K_{S 0}$ & $K$ & $K^{\mathrm{n}}$ & $E_{T R}$ & $\gamma_{0}$ & $n$ & $\Theta_{D}$ & $\beta_{0}$ & $\gamma_{e}$ \\
\hline $\mathrm{FeS}(\mathrm{s})$ & $5340^{\mathrm{a}}$ & $117.8^{\mathrm{a}}$ & $4.1^{\mathrm{a}}$ & $-0.0339^{\mathrm{a}}$ & $0.26^{\mathrm{a}}$ & $1.54^{\mathrm{a}}$ & $1.0^{\mathrm{a}}$ & $674^{\mathrm{b}}$ & $0.25^{\mathrm{c}}$ & $1.34^{\mathrm{d}}$ \\
$\mathrm{FeS}(\mathrm{l})$ & $5150^{\mathrm{a}}$ & $98.9^{\mathrm{a}}$ & $4.95^{\mathrm{a}}$ & $-0.05805^{\mathrm{a}}$ & $0.90^{\mathrm{a}}$ & $1.40^{\mathrm{a}}$ & $1.0^{\mathrm{a}}$ & $0^{\mathrm{e}}$ & $0.05^{\mathrm{c}}$ & $1.34^{\mathrm{d}}$ \\
$\mathrm{FeS}$ & $5011^{\mathrm{f}}$ & $162^{\mathrm{f}}$ & $4.7^{\mathrm{f}}$ & $-0.03135^{\mathrm{f}}$ & $0.0^{\mathrm{f}}$ & $1.56^{\mathrm{f}}$ & $1.0^{\mathrm{f}}$ & $705^{\mathrm{g}}$ & 0.0 & $-0^{\mathrm{a}}$ \\
$\mathrm{E}-\mathrm{Fe}$ & $8298^{\mathrm{h}}$ & $170.5^{\mathrm{i}}$ & $5.25^{\mathrm{i}}$ & $-0.0263^{\mathrm{i}}$ & $0.139^{\mathrm{j}}$ & $2.2^{\mathrm{k}}$ & $1.62^{\mathrm{k}}$ & $385^{1}$ & $0.092^{\mathrm{d}}$ & $1.34^{\mathrm{d}}$ \\
\hline
\end{tabular}

a Brown et al. [1984].

b Svendsen et al. [1989].

c Fit to present data.

d Assumed (Boness et al. [1986]).

e Assumed.

f Ahrens and Jeanloz [1987]. g Fit to tabulation from Chase et al. [1985].

h Mao et al. [1990].

i Fit to Hugoniot using stated value of $\rho_{0}$.

j Chase et al. [1985].

k Jeanloz [1979].

1 Andrews [1973].

$$
\left(\frac{\partial \ln K}{\partial \ln \rho}\right)_{T}=2 n+3 \gamma-\frac{1}{3}
$$

In the case of $\mathrm{LiF}$, we can check this model against the measured variation of $K$ on the $298 \mathrm{~K}$ isotherm. Using the properties from Table 5 , we predict that at STP, $(\partial \ln K / \partial \ln \rho)_{T}=7.795$, in good agreement with the experimental value of 7.9 [Andersson and Bäckström, 1987]. The result we obtain for $\mathrm{Al}_{2} \mathrm{O}_{3}$ is a factor of $\sim 10$ lower than is obtained from the model used by Bass et al. [1987], in agreement with the findings of Gallagher et al. [1994]. For the densities of the windows at 1 bar and elevated temperatures, we use the thermal expansion expression of Saxena and Shen [1992] for $\mathrm{Al}_{2} \mathrm{O}_{3}$ and that of Touloukian et al. [1977] for LiF. We assume entropies of melting of 933 $\mathrm{J} / \mathrm{kg} \mathrm{K}$ and $468 \mathrm{~J} / \mathrm{kg} \mathrm{K}$ for $\mathrm{Al}_{2} \mathrm{O}_{3}$ and $\mathrm{LiF}$, respectively [Tan and Ahrens, 1990].

At STP, both $\mathrm{FeS}_{2}$ and stoichiometric $\mathrm{FeS}$ are semiconductors. FeS changes to a metallic phase at $3.4 \mathrm{GPa}$ upon room temperature compression [King and Prewitt, 1982] and should continue to behave as a metal at the higher pressures considered in our study. Although the electrical properties of $\mathrm{FeS}_{2}$ have apparently not been determined at high pressures, both the temperature variation of the band gap of $\mathrm{FeS}_{2}$ [Karguppikar and Vedeshwar, 1988] and the general decreases of the band gaps of semiconductors under compression [Harrison, 1989] suggest that it should also behave as a metallic conductor under the conditions achieved in our experiments. Thus the thermal conductivities of both are dominated by the conduction electrons and may be estimated via the Wiedemann-Franz relation [Kittel, 1966]:

$$
K=L \sigma T,
$$

where $L$ is the Lorentz number and $\sigma$ is the electrical conductivity. Typically, $L=2.45 \times 10^{-8} \mathrm{~W} \Omega / \mathrm{K}^{2}$, in good agreement with theory. Although Schloessin and Secco [1991] note that there are deviations from this behavior, the deviations are serious only in ferromagnetic phases. Otherwise, $L$ seems to fall within $10 \%$ of the theoretical value, which is quite satisfactory for the present case. There are no experimental data for $\sigma$ in the iron sulfides under the conditions of interest. For a random (metallic) mixture of $\mathrm{Fe}$ and $\mathrm{S}, \sigma$ can be estimated by [Mott and Jones, 1959]

$$
\frac{1}{\sigma}=\frac{1-\chi_{S}}{\sigma_{F e}}+\frac{\chi_{S}}{\sigma_{s}}+B x_{S}\left(1-\chi_{s}\right)
$$

where $x_{S}$ is the atomic fraction of $S$ and $C$ typically falls in the range from $5 \times 10^{-7}$ to $5 \times 10^{-6} \Omega \mathrm{m}$. Because this formula generally underestimates $\sigma$ for a crystalline metal, we assign $B$ its minimum typical value of $5 \times 10^{-7} \Omega \mathrm{m}$. The value of for $\varepsilon-F e$ an the $298 \mathrm{~K}$ isotherm can be approximated by a fit to the temperature-corrected shock wave data of Keeler and Royce [1971], where

$$
\sigma_{F e}=a_{F e}+b_{F e}\left(\rho / \rho_{o}\right) .
$$

Here, $\rho_{0}$ is the STP density of $\varepsilon-F e$, taken as $8.298 \mathrm{Mg} / \mathrm{m}^{3}$ [Mao et al., 1990], $a_{F e}=-5.1826 \times 10^{8} \Omega^{-1} \mathrm{~m}^{-1}$ and $b_{F e}=$ $5.2788 \times 10^{8} \Omega^{-1} \mathrm{~m}^{-1}$. We assume that $\sigma_{S}$ obeys a similar relation to (34) with $a_{S}$ and $b_{S}$ proportional to $a_{F e}$ and $b_{F e^{\prime}}$ using $\left(\sigma_{S} / \sigma_{F e}\right)_{1 \text { bar }}$ as the proportionality constant. The value of $\sigma_{S}$ at STP is taken by assuming that (33) applies to metallic $\mathrm{FeS}_{2}$ and using the limiting value of $\sigma_{\mathrm{FeS}_{2}}$ as the band gap vanishes $\left(\sigma_{\mathrm{FeS}}=2.973 \times 10^{6} \Omega^{-1} \mathrm{~m}^{-1}\right.$ [Karguppikar and Vedeshwar, 1988]). The resulting values for $S$ are $a_{S}=-3.3882 \times 10^{8} \Omega^{-1} \mathrm{~m}^{-1}$ and $b_{S}=3.4736 \times 10^{8} \Omega^{-}$ ${ }^{1} \mathrm{~m}^{-1}$. Correction to high temperatures is made by noting that [Mott and Jones, 1959]

$$
\sigma \propto e^{\theta_{E} / T}-1
$$

For $\Delta S_{m, s}$, we use the FeS 1 bar value of $1.29 k$ per atom [Chase et al., 1985]. For $C_{s,}$ we use the Dulong-Petit high temperature limit of $3 k$ per atom.

For models I and III, we can estimate the fraction $\chi_{s}$ of the sample initially melted by the shock and release or reshock processes by interpolating linearly in pressure along the melting curve from $\boldsymbol{\chi}_{s}=\mathbf{0}$ at the intersection with the solid Hugoniot to $x_{s}=1$ at the intersection with the liquid Hugoniot. These intersections are estimated from our experimental results and the equation of state studies of Brown et al. [1984] and Ahrens and Jeanloz [1987].

\section{Hugoniot Temperatures and Thermal Properties}

When the data are evaluated with (13)-(20), the Hugoniot and melting temperatures presented in Table 3 and Figure 8 are obtained. Comparison with the values of $T_{I}$ in Table 3 indicates that the corrections from $T_{l}$ are small $(\sim 0-1 \%)$ compared to the magnitude of $T_{I}$ itself, indicating that knowledge of the exact values of the properties incorporated into the foregoing calculations is unnecessary. In fact, the 

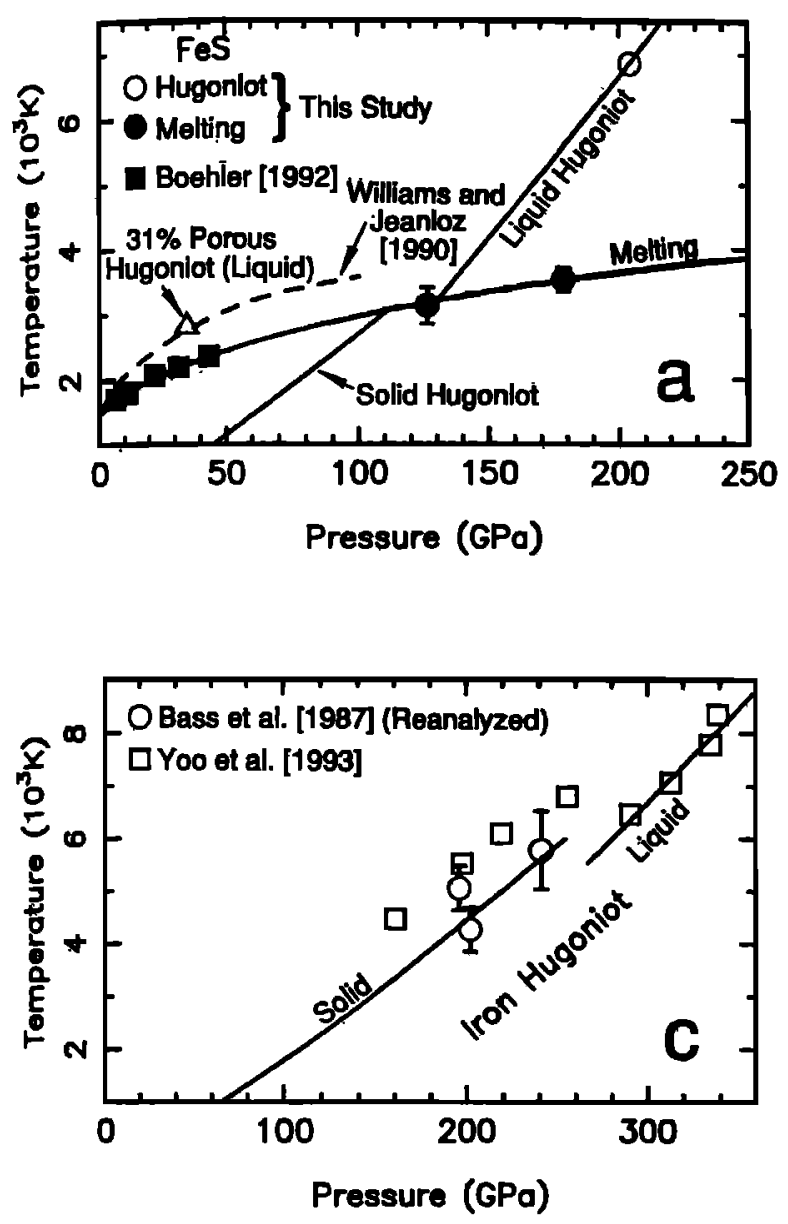

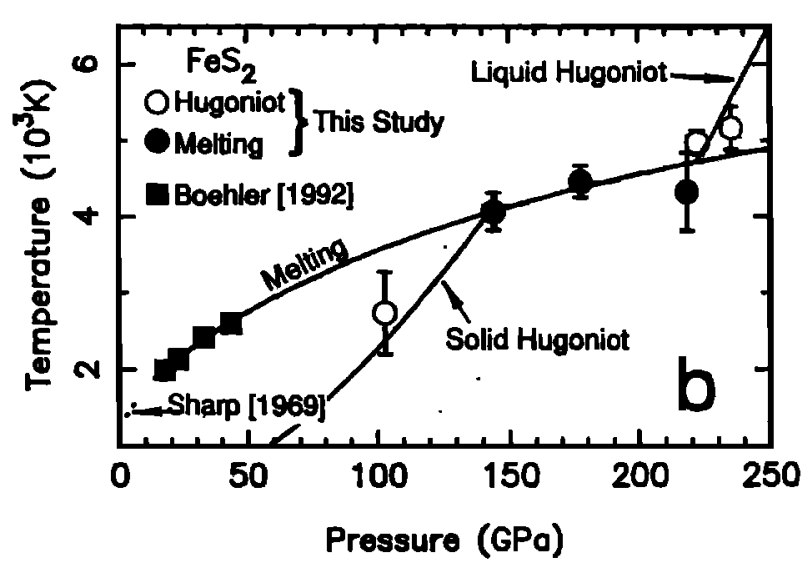

Iron Melting

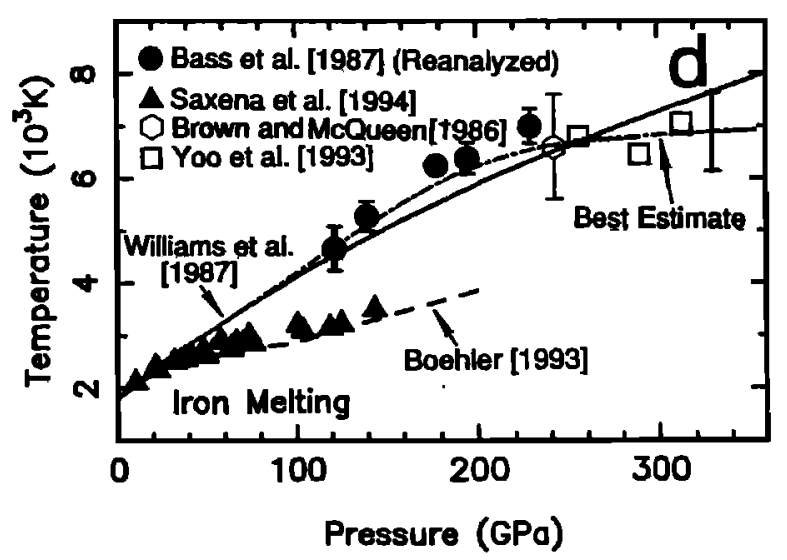

Figure 8. (a) Hugoniot and melting temperatures for FeS. The melting curve is fit to the present results and those of Boehler [1992]. The Hugoniot curves are calculated using the thermodynamic properties in Table 5. (b) Hugoniot and melting temperatures for $\mathrm{FeS}_{2}$, fit to the data and calculated from parameters in Table 5. (c) Hugoniot and melting temperature data for Fe. (d) Melting data for iron from static and dynamic experiments.

relative conductivities of the samples and windows, combined with the buffering latent heat effect, causes $T_{I}$ to be equal to $T_{m, s}$ when models I or III apply. The Hugoniot temperatures obtained in experiments 206 and 723 for FeS and 211, 216, and 217 for $\mathrm{FeS}_{2}$ can be used to constrain the specific heats of these materials at high pressures. These results should be treated with some caution because, unlike the melting results to be discussed in the next section, Hugoniot temperatures are very sensitive to the initial conditions of the sample, so that the measured Hugoniot temperatures are upper bounds to the actual values to be obtained from a perfectly nonporous sample. In our study, the results give us confidence that the measured values are close to the those representing nonporous samples. The temperatures of liquid FeS under shock compression show that $C_{V}>3 R$, indicating that electronic contributions cannot be ignored. If we assume the Sommerfeld form for the electronic specific heat [Boness et al., 1986],

$$
C_{e}=\beta T=\beta_{0}\left(\rho_{0} / \rho\right)^{\gamma_{e}} T
$$

and if we assume the same dependences on $\rho$ as found for $\mathrm{Fe}$ by Boness et al. [1986], then $\beta_{0}=0.05 \mathrm{~J} \mathrm{~kg}^{-1} \mathrm{~K}^{-2}$ and $\gamma_{e}=$
1.34 for liquid FeS. This assumes that the "lattice" contribution to $C_{V}$ is $3 k$ per atom. If as is commonly the case for liquids, the "lattice" contribution is less than $3 k$ per atom, then $\beta_{0}$ will be higher than the value given here. Although no direct observations of $\mathrm{FeS}$ in the solid state were made, consideration of the melting curve and the results of Brown et al. [1984] for the pressure range of shock melting allows us to obtain $\beta_{0}=0.25 \pm 0.10 \mathrm{~J} \mathrm{~kg}^{-1} \mathrm{~K}^{-2}$ for the high pressure solid phase of $\mathrm{FeS}$, assuming $\gamma_{e}=1.34$. Based on the temperature offset of the solid and liquid portions of the principal Hugoniot, we estimate that the entropy of melting of FeS is $\Delta S_{m, F e S}=203 \mathrm{~J} \mathrm{~kg}^{-1} \mathrm{~K}^{-1}$ at $120 \mathrm{GPa}$. An important point to be made here is the uncertainty and the value of $T_{I}$, and hence the Hugoniot temperature, for shot 206 . While the formal uncertainty on $T_{I}$ is small in a given fit, the large variation in $T_{I}$ obtained for different emissivity models suggests that, in reality, $T_{I}$ is poorly known for shot 206. Hence the results given here that depend on the Hugoniot temperature for shot 206 must also be treated as rather uncertain. By its same token, the variation in $T_{I}$ with choice of emissivity model for the other experiments is typically smaller than the formal fit uncertainties, so that the other results in this study can generally be considered more reliable. 
For $\mathrm{FeS}_{2}$, we have a direct measurement of the Hugoniot temperature $T_{H}$ in the solid. In contrast to the FeS results, we find that $C_{V} \ll 3 k$ per atom, indicating that either the Debye temperature for this phase is very high at high pressures or that some of the vibrational modes in the solid, perhaps those associated with $S$ atoms, are restricted. The latter might be the case if the $S_{2}$ units that exist at low pressure are still tightly bound at high pressures in the solid. Using this assumption, we take the effective DulongPetit value for $\mathrm{FeS}_{2}$ as $415.8 \mathrm{~J} \mathrm{~kg}^{-1} \mathrm{~K}^{-1}$ at high pressure. Caution should be used in interpreting this result, since it is based on only one data point with a large uncertainty. Although experiments 216 and 217 place constraints on the liquid phase of $\mathrm{FeS}_{2}$, the data scatter and lack of a separate equation of state for this phase, in particular the energy of transition from the solid to the liquid at STP, make $C_{V}$ difficult to determine. If we assume that $C_{V}$ for the liquid is similar to the solid phase value, then we find that the entropy of melting of $\mathrm{FeS}_{2}$ at $220 \mathrm{GPa}$ is $\Delta S_{m, F e S_{2}}=180 \mathrm{~J}$ $\mathrm{kg}^{-1} \mathrm{~K}^{-1}$.

Concerns might be raised about the effects of uncertainties in the initial densities on the amount of shock heating in the thin film samples used in most of these experiments [Nellis and Yoo, 1990]. Although the densities of the films are not directly measured, experience with $\mathrm{Fe}$ [Bass et al., 1987; Ahrens et al., 1990a] indicates that the bulk densities are close to the crystal densities. This is also demonstrated by the fact that the $T_{H}$ values are lower than expected for liquid $\mathrm{FeS}$, on the assumption that $C_{V}=$ $3 k / a t o m$. In any case our results for the melting of the samples are unaffected, because of the nature of the final state that we observe. Because the thin film samples are $\sim 1$ $\mu \mathrm{m}$ thick, reverberation of the shock wave has brought the sample to a pressure characteristic of the Hugoniot impedance match between the driver and the window by the time the first datum is obtained $-10 \mathrm{~ns}$ after arrival of the shock wave at the sample-window interface. Thus the pressure of the observed state is independent of the properties of the sample. Combining this and the fact that we are observing a temperature that is buffered by a large latent heat, we can know with certainty both the temperature and the pressure of the state we are observing and, since this state is on the melting curve, the melting temperature at the observed pressure.

Concems could also be raised about possible changes in the radiative transfer properties of the windows upon passage of the shock wave [Nellis and Yoo, 1990]. As mentioned earlier, the assumption that $\mathrm{Al}_{2} \mathrm{O}_{3}$ remains transparent under shock compressions has been called into question by Kondo [1994], who detects visible, probably nonthermal, radiation from shocked sapphire. This radiation would contaminate the signal from the sample and also indicated that the sapphire should be absorbing, and thus able to attenuate the interface radiation. Experimental data from McQueen and Isaak [1990] and the reanalysis of the data of Urtiew [1974] by Ahrens et al. [1990b], however, indicate that $\mathrm{Al}_{2} \mathrm{O}_{3}$ remains transparent upon being shocked to at least $200 \mathrm{GPa}$. In the present study, we have, with the exception of shot 217 , chosen the earliest time available after arrival of the shock wave at the interface. The thickness of shocked window material in the optical path is only $5-15 \mu \mathrm{m}$. Furthermore, the fact that we obtain different shock temperatures for different materials at similar window shock pressures strongly supports the conclusion that the radiation we observed is derived from the sample, not the window. Wise and Chhabildas [1986] demonstrate that $\mathrm{LiF}$, which was used in experiment 723 , remains transparent when shocked to at least $160 \mathrm{GPa}$. Figure 9 shows the deconvolved signals (voltage vs. time) for each channel in shot 203, which is fairly representative of the noise level and qualitative behavior. The time variation seen after arrival of the shock wave at the sample-window interface may be attributed to some combination of diffusion of heat from the driver-sample interface and optical effects in the shocked window. However, on the timescale of the measurement (the first 10 ns of data), the variation is relatively small, so that these effects do not introduce significant errors in the resulting temperature fits.

One of the greatest sources of potential error would be any undetected porosity in the thin films. As noted earlier, no porosity was detected, but if present, the large PV term in the energy would cause us to observe anomalously high single-phase Hugoniot temperatures. However, it must be stressed that this error would affect only the single-phase Hugoniot temperatures, not the melting temperatures. The reason, as has already been stated, is that the observed pressure is characteristic of the impedance match between the driver plate and the window, while the melting temperature in a metallic or predominantly ionic material is buffered by the latent heat and must fall on the melting curve at the observed pressure. This is an inescapable result. There is the possibility of kinetic inhibition of the phase transformation with superheating, but for materials as simple as metals and metallic sulfides, this is very unlikely. The conclusion is that the melting temperatures obtained in this study should be as reliable as the stated uncertainties indicate. The reliabilities of the single-phase Hugoniot temperatures are necessarily somewhat lower.

\section{Melting Curves in the Fe-S System}

The difficulty in obtaining usable samples has resulted in a very sparse data set for FeS. This material has also been studied by Williams and Jeanloz [1990] and Boehler [1992]. Figure 8a presents our data for melting, along with the melting curve of Williams and Jeanloz [1990] and the melting data of Boehler [1992].

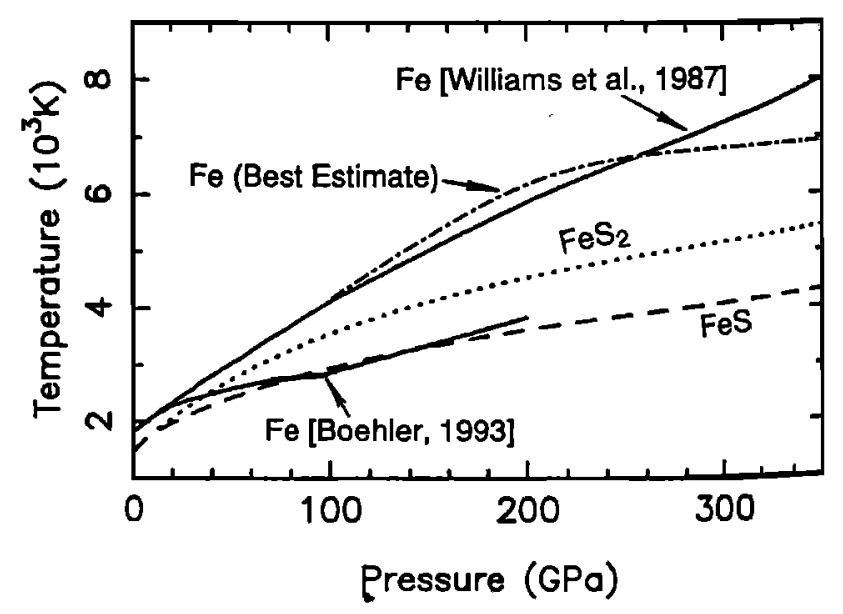

Figure 9. Melting curves for $\mathrm{FeS}$ and $\mathrm{FeS}_{2}$ compared to Fe melting curves. 
For extrapolation to higher pressures, we use the Lindemann law [Lindemann, 1910], which relates the temperature of melting, $T_{m}$, to the density of the solid at melting, $\rho_{m}$, via the relation [Gilvarry, 1956]

$$
\frac{d \ln T_{m}}{d \ln \rho}=2\left(\gamma_{L}-\frac{1}{3}\right)
$$

This expression is based on the assumption that the structure of a single solid phase is invariant along the melting curve. Usually, $\gamma_{L}$ is taken to be the lattice Grineisen parameter and is assumed to obey (24). To fit our data, we used the equation of state from Brown et al. [1984] and our results for $C_{V}$ (Table 5) to obtain the $\rho-T$ curve from the $P-T$ curve. In fitting parameters for the Lindemann melting relation, we considered data from both Williams et al. [1990] and Boehler [1992]. Both the general trends established by the data sets in comparison to the present results and the formal fits indicate that the data of Boehler [1992] are more consistent with our results. We combine his data set with ours and obtain for the melting of the high-pressure phase of FeS $\gamma_{L}=1.174 \pm 0.132$ and $n_{L}=$ $0.54 \pm 0.53$ at $\rho=5340 \mathrm{~kg} / \mathrm{m}^{3}$. The melting temperature at $\rho=5340 \mathrm{~kg} / \mathrm{m}^{3}$ is $1772 \pm 83 \mathrm{~K}$. We emphasize that these fit parameters are only valid when used with the equation of state parameters given in Table 5. The derived $P-T$ melting curve should be robust, however, since the fit is based on data obtained in the $P-T$ plane. This curve gives melting temperatures of $3240 \pm 200 \mathrm{~K}$ at $136 \mathrm{GPa}, 4210 \pm 700 \mathrm{~K}$ at the inner core boundary (ICB) pressure of $330 \mathrm{GPa}$, and $4310 \pm 750 \mathrm{~K}$ at $360 \mathrm{GPa}$.

Figure $8 \mathrm{~b}$ presents the data for our melting data for $\mathrm{FeS}_{2}$, along with those of Boehler [1992] and Sharp [1969]. To fit the Lindemann relation, we used the equation of state parameters (Table 5) obtained for pyrite by Ahrens and Jeanloz [1987] in conjunction with the specific heat discussed above. Again, the $P-T$ projection of the melting curve is not sensitive to inaccuracies in the equation of state parameters for $\mathrm{FeS}_{2}$ because the curve is anchored to data that occur in $P-T$ space, not $\rho-T$ space. We find values of $\gamma_{L}$ $=2.178 \pm 0.315$, and $n_{L}=1.63 \pm 0.68$, and $T_{m}=1598 \pm 240 \mathrm{~K}$ at $\rho=5011 \mathrm{~kg} / \mathrm{m}^{3}$. This value for $\gamma_{L}$ is larger than the value of the thermodynamic Grüneisen parameter obtained on the assumption that $n=1$ [Ahrens and Jeanloz, 1987]. It should actually be comparable or smaller if it truly represents the lattice value $\gamma_{L}$ as postulated in the original form of the Lindemann relation, but the difference is most probably explained by the different values of $n$, which was constrained to be 1 by Ahrens and Jeanloz [1987] but was left as a free parameter in the Lindemann fit. The resulting melting curve is significantly steeper than the FeS curve (Figure 9), with $T_{m}=3990 \pm 300 \mathrm{~K}$ at $136 \mathrm{GPa}, 5310 \pm 700 \mathrm{~K}$ at $330 \mathrm{GPa}$, and $5440 \pm 750 \mathrm{~K}$ at $360 \mathrm{GPa}$. It should be noted that at pressures below 6.4-8 GPa, the curve is a decomposition curve, rather than a melting curve [Sharp, 1969; Boehler, 1992].

Because our models for the thermal conductivities of $\mathrm{LiF}$ and $\mathrm{Al}_{2} \mathrm{O}_{3}$ differ substantially from those of Bass et al. [1987], we have reanalyzed their shock temperature data for Fe (Table 6). Some of the interface temperatures are quite high and can be rejected as being affected by sample porosity or excessive heat deposition at the sample-window interface. Based on the shock and release pressures achieved relative to the pressures of the solid-liquid mixed-phase region of the Hugoniot determined by Brown and McQueen [1986], the results of the diffusion equation solutions, and the trends established by the overall data set, we assign the melting and Hugoniot temperatures listed in Table 6 to the Bass et al. [1987] results. Also shown for comparison are the Hugoniot temperatures originally obtained by Bass et al. [1987]. The data we chose to use are presented in Figure 8d with the shock temperature data of Yoo et al. [1993], the melting curves of Williams et al. [1987] and Boehler [1993], and the diamond anvil cell melting data of Saxena et al. [1994]. None of the Bass et al. [1987] data can be reliably interpreted as giving a Hugoniot temperature in the liquid phase. The solid state Hugoniot temperatures are slightly lower than those obtained by Yoo et al. [1993].

The reanalyzed melting temperatures would indicate that the melting curve of $\mathrm{Fe}$ falls even higher than that of Williams et al. [1987]. We consider this unlikely because the resulting intersection of the solid state Hugoniot and the melting curve cannot be reconciled with the sound speed data of Brown and McQueen [1986]. While the explanation for this discrepancy is uncertain, it may be that the interface temperatures should be corrected slightly to account for nongray emissivity of $\mathrm{Fe}$ and for the effects of shock compression on the refractive indices of the window materials.

Substantial disagreement remains in the published melting curves for Fe. While we do not attempt to fully resolve this issue here, we do wish to analyze the implications of the different melting curves for the properties of solid Fe. Although there is disagreement on the melting temperatures, the sound speed data of Brown and McQueen [1986] are generally accepted to show that the solid Fe Hugoniot intersects the melting curve at $\sim 243 \mathrm{GPa}$ and that the completely liquid portion of the Hugoniot intersects the melting curve at $-270 \mathrm{GPa}$. Using a given $\boldsymbol{P}$ $V-E$ equation of state (Table 5), we can determine the electronic specific heat of the solid phase which would be required to give Hugoniot temperatures which agree with the melting curves at the intersection, under the assumption that the lattice specific heat is at the Dulong-Petit limit. The liquid $\mathrm{Fe}$ equation of state of Anderson and Ahrens [1994] gives the Hugoniot temperatures of the liquid which are in good agreement with the measured values from Yoo et al. [1993].

If we extrapolate the melting curve of Boehler [1993] to $-4250 \mathrm{~K}$ at $243 \mathrm{GPa}$, then $\beta_{0} \approx 0.286 \mathrm{~J} \mathrm{~kg}^{-1} \mathrm{~K}^{-2}$ for $\gamma_{e}=$ 1.34, which is a factor of $\sim 3$ greater than the theoretical value obtained by Boness et al. [1986]. On the other hand, the reanalyzed Bass et al. [1987] give $T_{m} \approx 7000 \mathrm{~K}$ at 243 $\mathrm{GPa}$, implying $\beta_{0} \approx 0.018 \mathrm{~J} \mathrm{~kg}^{-1} \mathrm{~K}^{-2}$, which is a factor of $\sim 5$ less than the theoretical value. The theoretical value of $\beta_{0}$ gives $T_{H}=5400 \mathrm{~K}$ at $243 \mathrm{GPa}$, which is consistent with the temperatures obtained by Yoo et al. [1993] if their two highest-pressure points on the solid Hugoniot are reanalyzed as giving melting temperatures in the final observed pressure state using the prescription of Tan and Ahrens [1990]. A melting curve based on the reanalyzed Bass et al. [1987] data in combination with data of Yoo et al. [1993] (Figure 8d) suggests a melting point of $6900 \pm 750 \mathrm{~K}$ for $\mathrm{Fe}$ at the inner core boundary pressure of $330 \mathrm{GPa}$. The 
Table 6. Reanalyzed Shock Temperature Data for Iron

\begin{tabular}{|c|c|c|c|c|c|}
\hline \multirow[t]{2}{*}{ Shot } & \multicolumn{2}{|c|}{ Pressure, GPa } & \multicolumn{3}{|c|}{ Temperature, $\mathbf{K}$} \\
\hline & Hugoniot & Final & Interface & Hugoniot ${ }^{\mathrm{a}}$ & Melting ${ }^{6}$ \\
\hline 167 & 196 & 157 & 4750 & $\begin{array}{c}5049 \\
(6110)\end{array}$ & \\
\hline 189 & 202 & 161 & 4010 & $\begin{array}{c}4263 \\
(5200)\end{array}$ & \\
\hline 188 & 241 & 189 & 5390 & $\begin{array}{c}5390 \\
(6870)\end{array}$ & \\
\hline 173 & 226 & 178 & 6240 & (7910) & 6240 \\
\hline 157 & 251 & 195 & 6380 & $(8200)$ & 6380 \\
\hline 168 & 300 & 229 & 6990 & (8930) & 6990 \\
\hline 190 & 227 & 122 & 4660 & $(6180)$ & 4660 \\
\hline 159 & 263 & 140 & 5270 & $(7240)$ & 5270 \\
\hline
\end{tabular}

a Values in parentheses are original values obtained by Bass et al. [1987].

b At final pressure.

Boehler [1993] and Saxena et al. [1994] results cannot be ruled out on the weight of the specific heat argument alone but is inconsistent with the measured Hugoniot temperatures and with the theoretical calculations.

\section{Discussion}

The present data provide important information on the phase relations in the Fe-S system, although the development of a detailed phase diagram requires the use of theoretical calculations and consideration of systematic variations in analog systems. Figure 10 shows the present results for $\mathrm{FeS}$ and $\mathrm{FeS}_{2}$ in comparison to the melting curve of $\mathrm{Fe}$, as determined by Williams et al. [1987]. At low pressures, $\mathrm{FeS}_{2}$ undergoes peritectic decomposition (i.e., incongruent melting) to pyrrhotite $\left(\mathrm{Fe}_{1-\mathrm{x}} \mathrm{S}\right)$ and a sulfur-rich liquid. However, the present results indicate that the pyrite melting/decomposition curve crosses the melting curve of $\mathrm{FeS}$ in the pressure range 10-15 $\mathrm{GPa}$, suggesting that the $\mathrm{FeS}-\mathrm{FeS}_{2}$ subsystem phase diagram undergoes a fundamental change below this pressure, including transition to congruent melting by $\mathrm{FeS}_{2}$, as suggested by Sharp [1969].

A consequence of the behavior of the $\mathrm{FeS}-\mathrm{FeS}_{2}$ subsystem is that we now see that the E-Fe-S phase diagram proposed by Anderson et al. [1989] is no longer tenable and that the eutectic behavior found by Williams and Jeanloz [1990] continues throughout the pressure range investigated. This is because, even in the $\gamma-\mathrm{Fe} P-T$ stability field, the Anderson et al. [1989] model would lead us to expect that $\mathrm{FeS}_{2}$ continues to melt at temperatures lower than does $\mathrm{FeS}$. Since the present data indicate that this is not the case, we conclude that a model calling for complete solid solution between $\mathrm{S}$ and $\mathrm{Fe}$ is inconsistent with the data. We must assume eutectic behavior for the Fe-S system throughout the pressure range of the core. The fact that the FeS melting curve falls well below those of either $\mathrm{Fe}$ or $\mathrm{FeS}_{2}$ may in fact be an indication that FeS could eventually become unstable as a congruent melting phase and undergo peritectic decomposition at high pressures.

\section{Summary}

New shock temperature data for $\mathrm{FeS}$ and $\mathrm{FeS}_{2}$ are interpreted in terms of their melting behavior at core pressures. The data show that at $P>15 \mathrm{GPa}, \mathrm{FeS}_{2}$ melts at higher temperatures than FeS.

Using a Lindemann law extrapolation with published equations of state of $\mathrm{FeS}$ and $\mathrm{FeS}_{2}$, we get $\gamma_{L}=$ $1.174 \pm 0.132, n_{L}=0.54 \pm 0.53$, and $T_{m}=1772 \pm 83 \mathrm{~K}$ at $\rho=$ $5340 \mathrm{~kg} / \mathrm{m}^{3}$ for FeS and $\gamma_{L}=2.178 \pm 0.315, n_{L}=$ $-1.63 \pm 0.68$, and $T_{m}=1598 \pm 240 \mathrm{~K}$ at $\rho=5011 \mathrm{~kg} / \mathrm{m}^{3}$ for $\mathrm{FeS}_{2}$. The melting curves thus described give $T_{m}=$ $3240 \pm 200 \mathrm{~K}$ at $136 \mathrm{GPa}, 4210 \pm 700 \mathrm{~K}$ at $330 \mathrm{GPa}$, and $4310 \pm 750 \mathrm{~K}$ at $360 \mathrm{GPa}$ for $\mathrm{FeS}$ and $3990 \pm 300 \mathrm{~K}$ at 136 $\mathrm{GPa}, 5310 \pm 700 \mathrm{~K}$ at $330 \mathrm{GPa}$, and $5440 \pm 750 \mathrm{~K}$ at $360 \mathrm{GPa}$ for $\mathrm{FeS}_{2}$. The entropies of melting are $203 \mathrm{~J} \mathrm{~kg}^{-1} \mathrm{~K}^{-1}$ for FeS and $180 \mathrm{~J} \mathrm{~kg}^{-1} \mathrm{~K}^{-1}$ for $\mathrm{FeS}_{2}$. Based on the Hugoniot and melting temperatures, we estimate that the Sommerfeld constant for the electronic specific heat at STP is $\beta_{0}=$ $.0 .25 \pm 0.10 \mathrm{~J} \mathrm{~kg}^{-1} \mathrm{~K}^{-2}$ for the solid high-pressure phase of FeS and $\beta_{0} \approx 0.05 \mathrm{~J} \mathrm{~kg}^{-1} \mathrm{~K}^{-2}$ for liquid FeS. This value for the liquid is based on the assumption that the "lattice" contribution to the specific heat is $3 k / a t o m$, which is an overestimate for most liquids. In contrast, the specific heat for solid $\mathrm{FeS}_{2}$ is only $\sim 2 k$ /atom, implying that tightly bonded $S_{2}$ structural units are important at high pressures.

A reassessment of the available shock wave melting data for $\mathrm{Fe}$ does not resolve the disagreement between different data sets but suggests that the Fe melting curve falls close to $6600 \pm 500 \mathrm{~K}$ at $243 \mathrm{GPa}$ and $6900 \pm 750 \mathrm{~K}$ at $330 \mathrm{GPa}$. The relative slopes and positions of the $\mathrm{Fe}, \mathrm{FeS}$, and $\mathrm{FeS}_{2}$ melting curves indicate that the Fe-S system continues to be dominated by eutectic behavior at high pressure and that $\mathrm{FeS}_{2}$, which undergoes peritectic decomposition at low pressure, melts congruently at high pressures. The fact that the FeS melting curve is much lower than that of $\mathrm{FeS}_{2}$ gives rise to the speculation that $\mathrm{FeS}$ may undergo peritectic decomposition to $\mathrm{FeS}_{2}$ and $\mathrm{Fe}$-rich liquid at high pressures.

Acknowledgments. We thank T. S. Duffy, W. J. Nellis, D. Heinz, and the Associate Editor for helpful comments on the manuscript: D. J. Stevenson and D. L. Anderson for much useful discussion; and E. Gello and $M$. Long for assistance in performing the experiments. We are grateful to T. Duffy and J. Hu for obtaining the FeS film $X$ ray diffraction data of Figure 2. Supported by NSF grant EAR 92-19906. Contribution 5156 of the Division of Geological and Planetary Sciences, California Institute of Technology. 


\section{References}

Ahrens, T. J., and R. Jeanloz, Pyrite: Shock compression, isentropic release, and composition of the Earth's core, J. Geophys. Res., 92, 10,363-10,375, 1987.

Ahrens, T. J., H. Tan, and J. D. Bass Analysis of shock temperature data for iron, High Pressure Res., 2, 145-157, 1990a.

Ahreas, T. J., J. D. Bass, and J. R. Abelson, Shock temperatures in metals, in Shock Compression of Condensed Matter-1989, edited by S. C. Schmidt, J. N. Johnson, and L. W. Davidson, pp. 851-857, Elsevier, New York, 1990b.

Anderson, W. W., and T. J. Ahrens, An equation of state for liquid iron and implications for the Earth's core, J. Geophys. Res., 99, 42734284, 1994.

Anderson, W. W., T. J. Ahrens, and B. Svendsen, Melting in the Fe-FeS system and its relation to the compositions of the core of Earth and Mars (abstract), Lunar Planet. Sci., XVIII, 21-22, 1987.

Anderson, W. W., B. Svendsen, and T. J. Ahrens, Phase relations in iron-rich systems and implications for the Earth's core, Phys. Earth Planet. Inter., 55, 208-220, 1989.

Andersson, S., and G. Bäckström, Thermal conductivity and heat capacity of single-crystal $\mathrm{LiF}$ and $\mathrm{CaF}_{2}$ under hydrostatic pressure, J. Phys. C Solid State Phys., 20, 5951-5962, 1987.

Andrews, D. J., Equation of state of the alpha and epsilon phases of iron, J. Phys. Chem. Solids, 34, 825-840, 1973.

Bass, J. D., B. Svendsen, and T. J. Ahrens, The temperatures of shockcompressed iron, in High Pressure Research in Mineral Physics, edited by M. Manghnani and Y. Syono, pp. 393-402, Terra Sci., Tokyo, 1987.

Boehler, R., The phase diagram of iron to $\mathbf{4 3 0} \mathrm{kbar}$, Geophys. Res. Lett. 13, 1153-1156, 1986.

Boehler, R., Melting of the Fe-FeO and the Fe-FeS systems at high pressure: Constraints on core temperatures, Earth Planet. Sci. Lett., III, 217-227, 1992.

Boehler, R., Temperatures in the Earth's core from melting-point measurements of iron at high static pressures, Nature, 363, 534-536, 1993.

Boehler, R., N. von Bargen, and A. Chopelas, Melting, thermal expansion, and phase transitions of iron at high pressures, $J$. Geophys. Res., 95, 21731-21736, 1990.

Boness, D. A., J. M. Brown, and A. K. McMahan, The electronic thermodynamics of iron under Earth core conditions, Phys. Earth Planet. Inter., 42, 227-240, 1986.

Born, M., and E. Wolf, Principles of Optics, 6th ed., 808 pp., Pergamon, Tarrytown, N.Y., 1980.

Baslough, M. B., Shock-wave properties and high-pressure equations of state of geophysically important materials, Ph.D. thesis, Calif. Inst. of Technol., Pasadena, 1984.

Bret, R., Chemical equilibration of the Earth's core and upper mantle, Geochim. Cosmochim. Acta, 48, 1183-1188, 1984.

Brett, R., and P. M. Bell, Melting relations in the Fe-rich portion of the system Fe-FeS at $30 \mathrm{~kb}$ pressure, Earth. Planet. Sci. Lett., 6, 479-482, 1969.

Britt, D. T., J. F. Bell, H. Haack, and E. R. D. Scott, The reflectance spectrum of troilite and the T-type asteroids (abstract), Meteoritics, 27, 207, 1992.

Brown, J. M., and R. G. McQueen, Phase transitions, Grüneisen parameter, and elasticity for shocked iron between $77 \mathrm{GPa}$ and 400 GPa, J. Geophys. Res., 91, 7485-7494, 1986.

Brown, J. M., T. J. Ahrens, and D. L. Shampine, Hugoniot data for pyrrhotite and the Earth's core, J. Geophys. Res., 89, 6041-6048, 1984.

Carslaw, H. S., and H. Jaeger, Conduction of Heat in Solids, 2nd ed., Oxford Univ. Press, New York, 1959.

Carter, W. J., Equation of state of some alkali halides, High Temp. High Pressure, 5, 313-318, 1973.

Chase, H. S., C. A. Davies, J. R. Downey, D. J. Frurip, R. A McDonald, and A. N. Syverud, JANAF thermochemical tables, 3rd ed., J. Phys. Chem Ref. Data, 14, suppl. 1, 1-1856, 1985.

Erskine, D., High pressure Hugoniot of sapphire, in Proceedings of 1993 AIRAPT/APS Conference, High-Pressure Science and Technology, pp. 141-143, Am. Inst. of Phys., New York, 1994.

Gallagher, K. G., J. D. Bass, T. J. Ahrens, M. Fitzner, and J. R. Abelson, Shock temperature of stainless steel and a high pressure-high temperature constraint on thermal diffusivity of $\mathrm{Al}_{2} \mathrm{O}_{3}$, in Proceedings of 1993 AIRAPT/APS Conference, High-Pressure
Science and Technology, pp. 963-966, Am. Inst. of Phys., New York, 1994.

Gilvarry, J. J., Grïneisen's law and the fusion curve at high pressures, Phys. Rev., 102, 317-325, 1956.

Grover, R., and P A. Urtiew, Thermal relaxation at interfaces following shock compression, J. Appl. Phys., 45, 146-152, 1974.

Harrison, W. A., Electronic Structure and the Properties of Solids: The Physics of the Chemical Bond, 586 pp., Dover, Mineola, N.Y., 1989.

Jeanloz, R., Properties of iron at high pressure and the state of the core, J. Geophys. Res., 84, 6059-6069, 1979.

Karguppikar, A. M., and A. G. Vedeshwar, Electrical and optical properties of natural iron pyrite $\left(\mathrm{FeS}_{2}\right)$, Phys. Status Solidi $A_{1} 109$, 549-558, 1988.

Keeler, R. N., and E. B. Royce, Electrical conductivity of condensed media at high pressures, in Physics of High Energy Density, edited by P. Caldirola and H. Knoepfel, Proc. Int. Sch. Phys. Enrico Fermi, 48, 106-125, 1971.

King, H. E., and C. T. Prewitt, High-pressure and high-temperature polymorphism of iron sulfide (FeS), Acta Crystallogr., Sect. B, 38, $1877-1887,1982$

Kittel, C., Introduction to Solid State Physics, John Wiley, New York, 1966.

Kondo, K., Window problem and complementary method for shocktemperature measurements of iron, in Proceedings of 1993 AIRAPT/APS Conference, High-Pressure Science and Technology, pp. 1555-1558, Am. Inst. of Phys., New York, 1994.

Kondo, K., and T. J. Ahrens, Heterogeneous shock-induced thermal radiation in minerals, Phys. Chem. Miner., 9, 173-181, 1983.

Lindemann, F. A., Uber die Berechnung molekularer Eigenfrenquenzen, Phys. Z., 11, 609-612, 1910.

Lyzenga, G. A., and T. J. Ahrens, A multi-wavelength optical pyrometer for shock compression experiments, Rev. Sci. Instrum. 50, 1421-1424, 1979.

Mao, H. K., Y. Wu, L. C. Chen, and J. F. Shu, Static compression of iron to $300 \mathrm{GPa}$ and $\mathrm{Fe}_{0.8} \mathrm{Ni}_{2}$ alloy to $260 \mathrm{GPa}$ : Implications for the composition of the core, J. Geophys. Res., 95, 21,737-21,742, 1990.

Marsh, S. P., LASL Shock Hugoniot Data. 658 pp., Univ. Calif. Press, Los Angeles, 1980.

McQueen, R. G., and D. G. Isaak, Characterizing windows for shock wave radiation studies, J. Geophys. Res., 95, 21,753-21,765, 1990.

Men', A. A., A. Z. Chechel'nitskii, V. A. Sokolov, and E. N. Simun, Thermal conductivity of lithium fluoride in the $300-1000^{\circ} \mathrm{K}$ temperature range, Sov. Phys. Solid State, Engl. Transl., 15, 1844 $1845,1974$.

Mitchell, A. C., and W. J. Nellis, Shock compression of aluminum, copper, and tantalum, J. Appl. Phys., 52, 3363-3375, 1981.

Mott, N. F., and H. Jones, The Theory of the Properties of Metals and Alloys, Dover, Mineola, N. Y., 1959.

Murthy, V. R., and H. T. Hall, The origin and composition of the Earth's core, Phys. Earth Planet. Inter., 6, 123-130, 1972.

Nellis, W. J., and C. S. Yoo, Issues concerning shock temperature measurements of iron and other metals, J. Geophys. Res., 95, 21749$21752,1990$.

Petrov, A. V., N. S. Tsypkina, and Y. A. Logachev, Temperature dependence of thermal conductivity in alkali halide salts at elevated temperatures, Sov. Phys. Solid State, EngL Transl. 16, 39-41, 1974.

Ringwood, A. E., Composition of the core and implications for origin of the Earth, Geochem. J., 11, 111-136, 1977.

Ringwood, A. E., and S. E. Kesson, Composition and origin of the moon, Proc. Lunar Sci. Conf., 8th, 371-398, 1977.

Roufosse, M. C., and R. Jeanloz, Thermal conductivity of minerals at high pressures: The effect of phase transitions, J. Geophys. Res., 88, 7399-7409, 1983.

Ruff, L., and D. L. Anderson, Core formation, evolution, and convection: A geophysical model, Phys. Earth Planet. Inter., 21, 181-201, 1980.

Sato, K., Reflectivity spectra and optical constants of pyrites (FeS $\mathrm{CoS}_{2}$, and $\mathrm{NiS}_{2}$ ) between 0.2 and $4.4 \mathrm{eV}, J$. Phys. Soc. Jpn., 53 , 1617-1620, 1984.

Saxena, S. K., and G. Shen, Assessed data on heat capacity, thermal expansion, and compressibility for some oxides and silicates, $J$. Geophys. Res., 97, 19,813-19,825, 1992.

Saxena, S. K., G. Shen, and P. Lazor, Temperatures in Earth's core based on melting and phase transformation experiments on iron, Science, 264, 405-407, 1994. 
Schloessin, H. H., and R. A. Secco, Transport properties of liquid iron up to $6 \mathrm{GPa}$, High Pressure Res., 7, 232-235, 1991.

Schmitt, D. R., and T. J. Ahrens, Temperatures of shock-induced shear instabilities and their relationship to fusion curves, Geophys. Res. Lett., 10, 1077-1080, 1983.

Sharp, W. E., Melting curves of sphalerite, galena, and pyrrhotite and the decomposition curve of pyrite between 30 and 65 kilobars, $J$. Geophys. Res., 74, 1645-1652, 1969.

Stevenson, D. J., Models of the Earth's core, Science, 214, 611-619, 1981.

Sumino, Y., and O. L. Anderson, Elastic constants of minerals, in CRC Handbook of Physical Properties of Rocks, vol. III, edited by R. S. Carmichael, pp. 39-138, CRC Press, Boca Raton, Fla., 1984.

Svendsen, B., W. W. Anderson, and T. J. Ahrens, Ideal Fe-FeS and FeFeO phase relations and the Earth's core, Phys. Earth Planet. Inter., 55, 154-186, 1989.

Tan, H., and T. J. Ahrens, Shock temperature measurement for metals, High Pressure Res., 2, 159-182, 1990.

Touloukian, Y. S., R. W. Powell, C. Y. Ho, and P. G. Klemens, Thermophysical Properties of Matter, vol. 2, Thermal Conductivity-Nonmetallic Solids, 1172 pp., Plenum, New York, 1970.

Touloukian, Y. S., R. K. Kirby, R. E. Taylor, and T. Y. R. Lee, Thermophysical Properties of Matter, vol. 13, Thermal Expansion-Nonmetallic Solids, 1658 pp., Plenum, New York, 1977.

Urtiew, P. A., Effect of shock loading on transparency of sapphire crystals, J. Appl. Phys., 45, 3490-3493, 1974.

Urtiew, P. A., and R. Grover, Temperature disposition caused by shock interactions with material interfaces, J. Appl Phys., 45, 140, 1974.

Usselman, T. J., Experimental approach to the state of the core, I, The liquidus relations of the $\mathrm{Fe}$-rich portion of the $\mathrm{Fe}-\mathrm{Ni}$-S system from 30 to $100 \mathrm{~kb}$, Am. J. Sci., 275, 278-290, 1975 a.
Usselman, T. J., Experimental approach to the state of the core, II, Composition and thermal regime, Am. J. Sci., 275, 291-303, 1975b.

Walsh, J. M., M. H. Rice, R. G. McQueen, and F. L. Yarger, Shoct. wave compressions of twenty-seven metals: Equations of state of metals, Phys. Rev., 108, 196-216, 1957.

Weast, R. C., and M. J. Astle (Eds.), CRC Handbook of Chemistry and Physics, 63rd ed., CRC Press, Boca Raton, Fla., 1982.

Williams, $Q$., and $R$. Jeanloz, Melting relations in the iron-sulfur systeme at ultrahigh pressures: Implications for the thermal state of the Earth, J. Geophys. Res., 95, 19,299-19,310, 1990.

Williams, Q., R. Jeanloz, J. Bass, B. Svendsen, and T. J. Ahrens, The melting curve of iron to 250 gigapascals: A constraint on the temperature at Earth's center, Science, 236, 181-182, 1987.

Wise, J. L., and L. C. Chhabildas, Laser interferometer measurements of refractive index in shock-compressed materials, in Shock Woves in Condensed Matter, edited by Y. M. Gupta, pp. 441-454, Plenum, New York, 1986.

Yoo, C. S., N. C. Holmes, M. Ross, D. J. Webb, and C. Pike, Shock temperatures and melting of iron at Earth core conditions, Phys. Rev. Lett., 70, 3931-3934, 1993.

T. J. Ahrens, Seismological Laboratory, MS 252-21, California Institute of Technology, Pasadena, CA 91125. (e-mail: tja@seismo.gps.caltech.edu)

W. W. Anderson, Department of Geology and Physics, Georgin Southwestern College, Americus, GA 31709. (e-mail wandersoe gswrs6k1.gsw.peachnet.edu)

(Received May 16, 1994; revised June 7, 1995;

accepted June 15, 1995.) 